\title{
The Jacobi orientation and the two-variable elliptic genus
}

\author{
MATTHEW ANDO \\ CHRISTOPHER P FRENCH \\ NORA GANTER
}

\begin{abstract}
Let $E$ be an elliptic spectrum with elliptic curve $C$. We show that the sigma orientation of the first author, Hopkins and Strickland [5] and Hopkins [24] gives rise to a genus of SU-manifolds taking its values in meromorphic functions on $C$. As $C$ varies we find that the genus is a meromorphic arithmetic Jacobi form. When $C$ is the Tate elliptic curve it specializes to the two-variable elliptic genus studied by Eguchi et al [15], Höhn [23] Krichever [28], Dijkgraaf et al [13] and Borisov and Libgober $[6 ; 7 ; 8]$. We also show that this two-variable genus arises as an instance of the $S^{1}$-equivariant sigma orientation.
\end{abstract}

$55 \mathrm{~N} 34$

\section{Introduction}

Stable homotopy theory singles out the Witten genus, also called the "sigma orientation", for special attention among elliptic genera. For example, it is uniquely characterized by the fact that it refines to a map of $E_{\infty}$ spectra

$$
\text { MString } \rightarrow \text { tmf, }
$$

from the bordism spectrum of Spin manifolds with trivialized second Chern class to the spectrum of "topological modular forms." This spectrum maps canonically and naturally to all elliptic spectra, so the Witten genus is in this sense "initial" among elliptic genera; see Hopkins [25; 24] and Ando, Hopkins and Strickland [5].

On the other hand, the work on orbifold elliptic genera has focused attention on the two-variable elliptic genus of Eguchi et al [15], Hirzebruch, Berger and Jung [22], Höhn [23] and Krichever [28]. This is the genus for which Dijkgraaf et al produced a product formula, expressing the elliptic genera of the symmetric product orbifolds $X^{n} / / \Sigma_{n}$ as a function of the elliptic genus of $X$ [13]. Borisov and Libgober have proved several results about this genus. For example, they give a mathematical proof of the product formula. Most strikingly, they produce a related "resolution of singularities" 
elliptic genus and prove a McKay correspondence result comparing their two genera $[6 ; 7 ; 8]$.

In this paper, we explain the relationship between the sigma orientation and the twovariable elliptic genus. We express the relationship two ways. The first involves the analysis of $\mathrm{MU}\langle 2 p\rangle$-orientations of [5], and gives new insight on the modularity properties of the two-variable genus. The second uses the sigma orientation in $S^{1}$ equivariant elliptic cohomology, and gives new insight on the "level $N$ genera".

Let $E$ be a homotopy-commutative even periodic ring spectrum, so $E$ is complexorientable, and

$$
G=\operatorname{spf} E^{0} \mathbb{C} P^{\infty}
$$

is a (commutative, one-dimensional) formal group over spec $\pi_{0} E$. Let $\mathrm{MU}\langle 2 p\rangle$ be the bordism spectrum of manifolds $M$ with complex tangent bundle and trivializations of $c_{1}, \ldots, c_{p-1}$, so MU $\langle 2\rangle=\mathrm{MU}$ and $\mathrm{MU}\langle 4\rangle=\mathrm{MSU}$. In [5], the authors show that, for $p \leq 3$, the set of maps of ring spectra (or genera, or orientations)

$$
\mathrm{MU}\langle 2 p\rangle \rightarrow E
$$

is isomorphic to the set of " $\Theta{ }^{p}$-structures" on $G$. (See Breen [9], Ando, Hopkins and Strickland [5] and Section 3.) Briefly, let $A$ be either an elliptic curve or a onedimensional formal group, and let $\mathcal{I}=\mathcal{I}_{A}(0)$ be the ideal of functions on $A$ vanishing at the identity. Let $\Theta^{0} \mathcal{I}=\mathcal{I}$, and, for $p \geq 1$, let $\Theta^{p} \mathcal{I}$ be the line bundle on $A^{p}$ given by the formulas

$$
\begin{aligned}
\left(\Theta^{1} \mathcal{I}\right)_{a} & =\frac{\mathcal{I}_{0}}{\mathcal{I}_{a}} \\
\left(\Theta^{2} \mathcal{I}\right)_{a, b} & =\frac{\mathcal{I}_{0} \mathcal{I}_{a+b}}{\mathcal{I}_{a} \mathcal{I}_{b}} \\
\left(\Theta^{3} \mathcal{I}\right)_{a, b, c} & =\frac{\mathcal{I}_{0} \mathcal{I}_{a+b} \mathcal{I}_{a+c} \mathcal{I}_{b+c}}{\mathcal{I}_{a} \mathcal{I}_{b} \mathcal{I}_{c} \mathcal{I}_{a+b+c}},
\end{aligned}
$$

where $a, b, c$ are points of $A$. A $\Theta^{p}$-structure on $A$ is a trivialization of $\Theta^{p} \mathcal{I}$ which is compatible with various bits of structure on $\Theta^{p} \mathcal{I}$. We write $C^{p}(A ; \mathcal{I})$ for the set of $\Theta^{p}$-structures on $A$. There are natural maps

$$
\delta: C^{p}(A ; \mathcal{I}) \rightarrow C^{p+1}(A ; \mathcal{I})
$$

which, in the case of the formal group $G_{E}$, correspond to the restriction of orientations

$$
\delta s: \mathrm{MU}\langle 2 p+2\rangle \rightarrow \mathrm{MU}\langle 2 p\rangle \stackrel{s}{\rightarrow} E .
$$


Notice that there are isomorphisms

$$
\begin{aligned}
\left(\Theta^{2} \mathcal{I}\right)_{a, b} & \cong \Theta^{1} \mathcal{I}_{a} \otimes \frac{\mathcal{I}_{a+b}}{\mathcal{I}_{b}} \\
\left(\Theta^{3} \mathcal{I}\right)_{a, b, c} & \cong \Theta^{2} \mathcal{I}_{a, b} \otimes \frac{\mathcal{I}_{a+c} \mathcal{I}_{b+c}}{\mathcal{I}_{a+b+c} \mathcal{I}_{c}} .
\end{aligned}
$$

As we discuss in Section 4, a section of the right tensor factor can be viewed as an element of $\mathcal{O}_{X}$, where, in the $\Theta^{3}$ case for example, $X \subset A^{3}$ is a subspace of

$$
\left\{(a, b, c) \in A^{3} \mid a+b+c \neq 0, c \neq 0, a+c \neq 0, b+c \neq 0\right\} .
$$

For example, if $C$ is an elliptic curve over a ring $R$, then we can take

$$
X=\widehat{C}^{2} \times(C \backslash 0),
$$

by which we mean the pullback of the formal scheme $\widehat{C}^{2}$ along

$$
(C \backslash 0) \rightarrow \operatorname{spec} R \text {. }
$$

The case of a formal group $G$ over $R$ is more subtle. In Section 4, we consider

$$
R_{1}=\underset{k}{\operatorname{colim}} \mathcal{I}^{-k} \mathcal{O}_{G}
$$

in terms of a coordinate on $G$ we have

$$
R_{1} \cong R((y))
$$

Let $G_{1}$ and $G_{\circ}$ be formal groups obtained by pulling back $G$ in the diagram

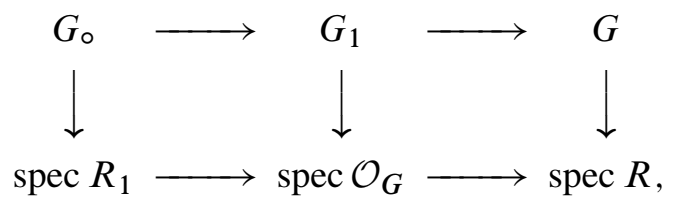

(we use $\circ$ to suggest the hole left over when the identity is removed from the $G$ in the base). Then we can use

$$
X=G_{\circ}^{2} .
$$

To see this, introduce a coordinate on $G$, and let $F$ be the resulting formal group law. Then

$$
\mathcal{O}_{X}=R((y))[[s, t]]
$$

and $y+{ }_{F} s$ and $y+{ }_{F} s+{ }_{F} t$ are units of $\mathcal{O}_{X}$. We show that a $\Theta^{2}$-structure on $G$ determines a $\Theta^{1}$-structure on $G_{\circ}$, and a $\Theta^{3}$-structure determines a $\Theta^{2}$-structure on $G_{\circ}$. 
As we explain in Section 5, if $R=\pi_{0} E$ and $G=G_{E}=\operatorname{spf} E^{0} \mathbb{C} P^{\infty}$, then

$$
R_{1}=\pi_{0} E^{\mathbb{C} P_{-\infty}^{\infty}}
$$

so in terms of orientations, we find that an SU-orientation

$$
t: \mathrm{MSU} \rightarrow E
$$

gives rise to an orientation

$$
t^{\sharp}: \mathrm{MU} \rightarrow E^{\mathbb{C} P_{-\infty}^{\infty}} .
$$

As the referee pointed out to us and as we explain in Example 5.7, there is a canonical complex orientation

$$
\mathrm{MU} \rightarrow \mathrm{MSU}^{\mathbb{C} P_{-\infty}^{\infty}}
$$

so that $t^{\#}$ is the composition

$$
\mathrm{MU} \rightarrow \mathrm{MSU}^{\mathbb{C P}} P_{-\infty}^{\infty} \stackrel{t}{\rightarrow} E^{\mathbb{C} P} P_{-\infty}^{\infty} .
$$

In any case, starting with a complex orientation $s: \mathrm{MU} \rightarrow E$, we obtain an orientation

$$
\delta s: \mathrm{MSU} \rightarrow E
$$

by restriction, and so a new orientation

$$
(\delta s)^{\sharp}: \mathrm{MU} \rightarrow E^{\mathbb{C P}} \boldsymbol{D}_{-\infty}^{\infty} .
$$

This procedure, applied to the Witten genus, produces the two-variable genus: let $K[[q]]$ be the spectrum representing complex $K$-theory with coefficients extended to $\mathbb{Z}[[q]$, and let

$$
\Phi: \mathrm{MU} \rightarrow K[[q]]
$$

be the complex orientation which associates to a manifold $M$ of complex dimension $d$ the genus

$$
\text { Todd }\left(M ; \bigotimes_{n \geq 1} \operatorname{Sym}_{q^{n}}\left(T-\mathbb{C}^{d}\right) \bigotimes_{n \geq 1} \operatorname{Sym}_{q^{n}}\left(\bar{T}-\mathbb{C}^{d}\right)\right) .
$$

Its $K$-theory Euler class is

$$
\Phi(u, q)=\left(1-u^{-1}\right) \prod_{n \geq 1} \frac{\left(1-q^{n} u\right)\left(1-q^{n} u^{-1}\right)}{\left(1-q^{n}\right)^{2}} ;
$$

it is a version of the Witten genus. ${ }^{1}$

\footnotetext{
${ }^{1}$ The relationship is analogous to the relationship between the Todd genus and the $\hat{A}$ genus. For example, the genus (1.1) coincides with the Witten genus on SU-manifolds.
} 
As we explain in Section 6, the orientation $(\delta \Phi)^{\#}$ sends a manifold $M$ of dimension $d$ to the genus

$$
\begin{aligned}
& \phi(M, y, q)=\Phi\left(y^{-1}, q\right)^{-d} \\
& \quad \times \operatorname{Todd}\left(M ; \bigotimes_{n \geq 1} \operatorname{Sym}_{q^{n}} T \bigotimes_{n \geq 1} \operatorname{Sym}_{q^{n}} \bar{T} \bigotimes_{n \geq 1} \Lambda_{-y q^{n}}(T) \bigotimes_{n \geq 1} \Lambda_{-y^{-1} q^{n}}(\bar{T})\right) .
\end{aligned}
$$

This is one of the standard formulas for the two-variable elliptic genus, and we give precise comparisons to formulas in [23, p 59] and [6, p 4].

Our approach to the two-variable genus gives a new account of its modularity, analogous to the account in [5] of the modularity of the Witten genus. Abel's Theorem, or the Theorem of the Cube, implies that an elliptic curve $C$ has a unique $\Theta^{3}$-structure $s(C)$. An isomorphism of formal groups

$$
\gamma: G \cong \widehat{C}
$$

then endows $G$ with the $\Theta^{3}$-structure $\left.\left(\gamma^{3}\right)^{*} s(C)\right|_{\widehat{C}^{3}}$. The data $(E, C, \gamma)$ comprise an elliptic spectrum, and the map of ring spectra

$$
s(E, C, \gamma): \mathrm{MU}\langle 6\rangle \rightarrow E
$$

arising from the $\Theta^{3}$-structure is called the sigma orientation.

For example, the Tate curve is a (generalized) elliptic curve $C_{\text {Tate }}$ over $\mathbb{Z}[[q]]$, equipped with an isomorphism

$$
\gamma_{\text {Tate }}: \widehat{\mathbb{G}}_{m} \cong \widehat{C}_{\text {Tate }}
$$

Now $\widehat{\mathbb{G}}_{m}$ is the formal group of complex $K$-theory, so $\left(K[[q]], C_{\text {Tate }}, \gamma_{\text {Tate }}\right)$ is an elliptic spectrum, denoted $K_{\text {Tate }}$ for short. In [9] and [5, Section 2.6,2.7], it is shown that $^{2}$

$$
s\left(C_{\text {Tate }}\right)=\delta^{2} \Phi \in \Theta^{3} \mathcal{I}_{C_{\text {Tate }}}(0) .
$$

Now observe that

$$
s\left(C_{\text {Tate }}\right)^{\#}=\left(\delta^{2} \Phi\right)^{\#}=\delta\left(\delta \Phi^{\sharp}\right): \mathrm{MSU} \rightarrow K[[q]]^{\mathbb{C} P_{-\infty}^{\infty},}
$$

so the two-variable elliptic genus of an SU-manifold is controlled by the canonical $\Theta^{3}$-structure on the Tate curve. This leads to a new proof of the (known) fact that the two-variable elliptic genus of an SU-manifold is a meromorphic Jacobi form. To give a precise statement, let $E \mathcal{J}_{C}$ be the cohomology theory formed by extending the coefficients of $E$ to $\Gamma\left(\mathcal{O}_{C \backslash 0}\right)$. In Section 7 we prove the following result.

\footnotetext{
${ }^{2}$ A generalized elliptic curve may have more than one $\Theta^{3}$-structure, but there is a unique rule $C \mapsto s(C)$ which is natural in $C$.
} 
1.4 Theorem An elliptic spectrum $(E, C, \gamma)$ determines a canonical orientation of SU-manifolds

$$
J_{(E, C, \gamma)}: \mathrm{MSU} \rightarrow E \mathcal{J}_{C} .
$$

The fact that the cubical structure on the Tate curve is of the form (1.3) implies that $J_{K_{\text {Tate }}}$ factors through $\mathrm{MU}$, and indeed the diagram

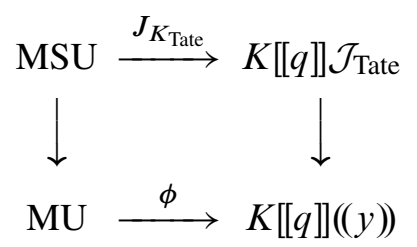

commutes, where $\phi$ is the genus (1.2).

We call $J$ the Jacobi orientation, because, as we explain in Theorem 7.7, the naturality of $J$ in the elliptic spectrum implies that the genus associated to $J_{K_{\text {Tate }}}$ takes its values in meromorphic Jacobi forms of index zero. We emphasize that $J_{(E, C, \gamma)}$ is canonically determined by the elliptic spectrum $(E, C, \gamma)$, and the variety of expressions for two-variable genera in the literature reflects choices in how to expand it.

Let $\mathbb{T}$ be the circle group. Jacobi forms also appear as elements of the $\mathbb{T}$-equivariant elliptic cohomology of spheres of representations. In Section 8, we give another formula for the two-variable elliptic genus in terms of the $\mathbb{T}$-equivariant sigma orientation. If $V$ is a complex vector bundle over $X$, let $V y^{-1}$ denote $V$, considered as $\mathbb{T}$-bundle via the inverse of the standard action of $\mathbb{T}$. We also write $y^{-1}$ for the trivial bundle, with the inverse of the standard action. Let $d=\operatorname{rank} T$, where $T$ is the complex tangent bundle of $X$, and let

$$
\xi=T y^{-1}-T-d y^{-1}=(T-d)\left(y^{-1}-1\right)-d .
$$

For $i \geq 0$ let $c_{i}^{\mathbb{T}}$ denote the Borel equivariant Chern classes. We note (Lemma 8.6) that

$$
c_{1}^{\mathbb{T}}(\xi)=0
$$

and if $c_{1}(T)=0$ then

$$
c_{2}^{\mathbb{T}}(\xi)=0 .
$$

It follows using [3;2] that if $c_{1}(T)=0$, then $\xi$ has an equivariant sigma orientation, which is a Thom class $U=U(\xi)$ in $E_{\mathbb{T}}\left(X^{\xi}\right)$, the $\mathbb{T}$-equivariant elliptic cohomology of Grojnowski associated to a complex elliptic curve $C$ of the form $\mathbb{C} /(2 \pi i \mathbb{Z}+2 \pi i \tau \mathbb{Z})$ (the first author and John Greenlees prove an analogous result for Greenlees's equivariant spectrum [18] in [4]). If we compose the Pontrjagin-Thom map

$$
S^{0} \rightarrow X^{-T}
$$


with the relative zero section

$$
X^{-T} \rightarrow X^{T y^{-1}-T}
$$

and then desuspend by $d y^{-1}$, we obtain a map

$$
g: S^{-d y^{-1}} \rightarrow X^{\xi}
$$

By pulling back the Thom class along this map we obtain an element of

$$
\Gamma E_{\mathbb{T}}\left(S^{-d y^{-1}}\right) \cong \Gamma \mathcal{I}_{C}(0)^{-d}
$$

We show (Proposition 8.15) that this is the two-variable elliptic genus. We are grateful to M Hopkins for suggesting this approach to the two-variable genus. The relationship between our two approaches is explained in Remark 8.7.

A similar argument accounts for the level $N$ elliptic genera of Hirzebruch [21], Witten [35] and Hirzebruch, Berger and Jung [22]. If instead of $c_{1}(T)=0$ we have $c_{1}(T) \equiv 0$ $\bmod N$, then $c_{2}^{\mathbb{T}[N]}(\xi)=0$. The work of [3;2] then shows that $\xi$ has an equivariant sigma orientation $U_{N} \in E_{\mathbb{T}[N]}\left(X^{\xi}\right)$. Pulling back this Thom class along $g$ as in (1.5) gives an element

$$
g^{*} U_{N} \in \Gamma E_{\mathbb{T}[N]}\left(S^{-d y^{-1}}\right) \cong \Gamma \mathcal{O}_{C[N]} .
$$

In Proposition 8.21, we show that the value of $g^{*} U_{N}$ at $a \in C[N]$ is just the level $N$ elliptic genus of $X$ evaluated at $a$.

In [17], the third author shows that $H_{\infty}$ elliptic genera have a product formula like that of [13]. Either of the accounts of the two-variable elliptic genus given here can be used to prove that the two-variable genus is $H_{\infty}$, once one knows that the Witten genus or sigma orientation from which it was constructed is $H_{\infty}$. We will return to that story at another time.

During their work on this paper, Ando was supported by NSF grant DMS-0306429, and Ganter was supported by NSF grant DMS-0504539. Some of the work took place while Ando and Ganter were visiting MSRI and Stanford University. We thank R Cohen for his hospitality. We thank A Ghitza, J Greenlees, J de Jong, M Hopkins, J Lurie, and H Sadofsky for useful conversations. We are grateful to the referee for catching a mistake in an earlier version of the paper and helping us to correct it, and for several helpful suggestions which improved the paper. We are responsible for the shortcomings which remain. 


\section{Notation}

\subsection{Groups}

We record the notation for some constructions which make sense in any setting where one has a notion of abelian group object $G$ over an object $S$, and where the line bundles over $X$ form a Picard category. Our examples will be elliptic curves and formal groups.

If $G$ is an abelian group over $S$, we write $0: S \rightarrow G$ for its identity section. If $I$ is a set, we write $G^{I}$ for the product $G_{S}^{I}$. If $f: I \rightarrow J$, then we write $\pi_{f}$ for the induced map

$$
\pi_{f}: G^{J} \rightarrow G^{I}
$$

If $I \subseteq J$ then we may abbreviate this as $\pi_{I}$, and we may even indicate $I$ by listing its elements. It will also be convenient to write $\hat{\pi}_{I}$ for $\pi_{J \backslash I}$. Thus if $J=\{1,2,3\}$ then $\pi_{13}$ and $\hat{\pi}_{2}$ indicate the same map $G^{3} \rightarrow G^{2}$. As a special case we have

$$
\pi_{\varnothing}=G^{J} \rightarrow S \text {. }
$$

We write

$$
\mu_{I}: G^{J} \stackrel{\pi_{I}}{\rightarrow} G^{I} \stackrel{\mu}{\rightarrow} G
$$

for projection to $G^{I}$ followed by multiplication. It is convenient to set $\mu_{\varnothing}=0: G^{J} \rightarrow$ $S \rightarrow G$.

\subsection{Change of base for formal groups}

If $G$ is a formal group over $S=\operatorname{spec} A$, and $T=\operatorname{spec} B \rightarrow S$ is an $S$-scheme, then we can form the pullback

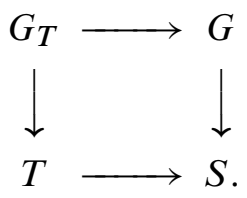

If $\mathcal{I}(0)$ denotes the ideal of the identity of $G$, then explicitly the ring of functions on $G_{T}$ is the completed tensor product

$$
\mathcal{O}_{G_{T}}=\left(B \otimes_{A} \mathcal{O}_{G}\right)_{\mathcal{I}(0)}^{\wedge}
$$

\subsection{Power series and Laurent series}

Let $R$ be a ring. Then $R[[x]]$ will denote the ring of power series in $x$, and $R((y))$ will denote the ring of finite-tailed Laurent series in $y$. In this paper we will need to consider rings like

$$
A=R((y))[[x]] .
$$


Note that this is different from

$$
B=R[[x]]((y))
$$

For example

$$
\begin{aligned}
& \sum_{n \geq 0} y^{-n} x^{n} \in A \\
& \sum_{n \geq 0} y^{-n} x^{n} \notin B .
\end{aligned}
$$

but

An important point is that series of the form

$$
y+o(x) \in A
$$

are invertible in $A$, since $y$ is a unit of $R((y))$.

Rings such as $R((y))[[x]]$ will arise in situations like the following. Let $G \cong \operatorname{spf} R[[x]]$ be a formal group over spec $R$. If $G^{\prime}$ denotes the formal group over $R((y))$ which is the pullback

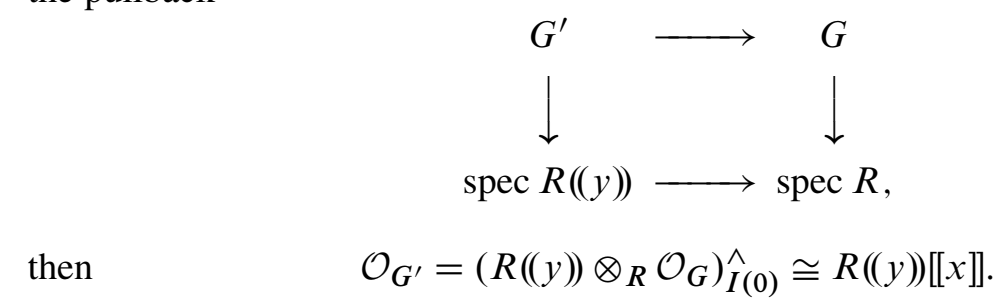

\subsection{Line bundles}

If $\left(X, \mathcal{O}_{X}\right)$ is some sort of ringed space, then a line bundle over $X$ will mean an invertible $\mathcal{O}_{X}$-module, and we write $\mathcal{L}^{\times}$for the associated $\mathbb{G}_{m}$-torsor of trivializations of $\mathcal{L}$; this participates in an equivalence of categories between the line bundles and $\mathbb{G}_{m}$-torsors over $X$.

\subsection{Vector bundles and Thom spectra}

If $X$ is a space, and $V$ is a vector bundle over $X$, then we write $X^{V}$ for the Thom spectrum

$$
X^{V} \stackrel{\text { def }}{=} \Sigma^{\infty}(D(V) / S(V)),
$$

where $D(V)$ is the disk bundle of $V$ and $S(V)$ is the sphere bundle. Notice that, if $\varepsilon$ denotes the trivial bundle of rank 1 over $X$, then

$$
X^{\varepsilon} \cong \Sigma \Sigma^{\infty} X_{+},
$$

Algebraic 83 Geometric Topology, Volume 8 (2008) 
and if $V / X$ and $W / Y$, then

$$
(X \times Y)^{V \oplus W} \cong X^{V} \wedge Y^{W} .
$$

The reason for using spectra rather than spaces is that one can extend the definition to virtual bundles. For example, if $V$ is a vector bundle over a finite complex, then there is a vector bundle $W$ over $X$ such that

$$
V \oplus W \cong N \varepsilon
$$

In view of (2.6) and (2.7), it is sensible to set

$$
X^{-V}=\Sigma^{-N} X^{W}
$$

and one shows that this stable homotopy type depends only on $V$. This definition extends to vector bundles over infinite complexes; see for example Lewis et al [29] and Rudyak [32].

We write $\mathrm{BU}\langle 2 p\rangle$ for the connective cover of $\mathbb{Z} \times \mathrm{BU}$ with its bottom nonzero homotopy group in degree $2 p$. So

$$
\begin{aligned}
& \mathrm{BU}\langle 0\rangle=\mathbb{Z} \times \mathrm{BU} \\
& \mathrm{BU}\langle 2\rangle=\mathrm{BU} \\
& \mathrm{BU}\langle 4\rangle=\mathrm{BSU} .
\end{aligned}
$$

We denote the associated Thom spectra by $\mathrm{MU}\langle 2 p\rangle$, so $\mathrm{MU}\langle 2\rangle=\mathrm{MU}$, and $\mathrm{MU}\langle 0\rangle=$ MP is the two-periodic complex cobordism spectrum.

\subsection{Cohomology}

Let $E$ be a homotopy commutative ring spectrum. If $X$ is a space, then $E^{*}(X)$ will denote its unreduced cohomology, which is a ring. If $Z$ is a spectrum, then $E^{*}(Z)$ will be its usual spectrum cohomology. Thus

$$
E^{*}(X)=E^{*}\left(\Sigma^{\infty} X_{+}\right) .
$$

With these conventions, a Thom isomorphism in $E$-cohomology for a vector bundle $V$ of rank $d$ over $X$ is an isomorphism of $E^{*}(X)$-modules

$$
E^{*}(X) \cong E^{*+d}\left(X^{V}\right)
$$

The reduced cohomology of a pointed space $X$ will be written $\widetilde{E}^{*}(X)$. 


\section{$3 \Theta^{p}$-structures}

We recall from $[9 ; 5]$ the notion of a $\Theta^{p}$-structure on a line bundle $\mathcal{L}$ over $G$. For $p \geq 1$ we define line bundles $\Theta^{p} \mathcal{L}$ over $G^{p}$ by the formulas

$$
\Theta^{p} \mathcal{L} \stackrel{\text { def }}{=} \bigotimes_{I \subseteq\{1, \ldots, p\}}\left(\mu_{I}^{*} \mathcal{L}\right)^{(-1)^{|I|}}
$$

Thus

$$
\begin{aligned}
\left(\Theta^{1} \mathcal{L}\right)_{a} & =\frac{\mathcal{L}_{0}}{\mathcal{L}_{a}} \\
\left(\Theta^{2} \mathcal{L}\right)_{a, b} & =\frac{\mathcal{L}_{0} \mathcal{L}_{a+b}}{\mathcal{L}_{a} \mathcal{L}_{b}} \\
\left(\Theta^{3} \mathcal{L}\right)_{a, b, c} & =\frac{\mathcal{L}_{0} \mathcal{L}_{a+b} \mathcal{L}_{a+c} \mathcal{L}_{b+c}}{\mathcal{L}_{a} \mathcal{L}_{b} \mathcal{L}_{c} \mathcal{L}_{a+b+c}} .
\end{aligned}
$$

We also define $\Theta^{0} \mathcal{L}=\mathcal{L}$, over $G$.

The formula (3.1) for $\Theta^{p}$ exhibits the symmetry of $\Theta^{p} \mathcal{L}$ over $G^{p}$. Precisely, we have the following.

3.2 Proposition (1) For $p>0, \Theta^{p}(\mathcal{L})$ is a rigid line bundle, that is, it comes with a trivialization of $0^{*} \Theta^{p}(\mathcal{L})$.

(2) For each permutation $\sigma \in \Sigma_{p}$, there is a canonical isomorphism

$$
\xi_{\sigma}: \pi_{\sigma}^{*} \Theta^{p}(\mathcal{L}) \cong \Theta^{p}(\mathcal{L}) .
$$

Moreover, these isomorphisms compose in the obvious way.

(3) There is a canonical identification (of rigid line bundles over $G_{S}^{p+1}$ )

(3.3) $\Theta^{p}(\mathcal{L})_{a_{1}, a_{2}, \ldots} \otimes \Theta^{p}(\mathcal{L})_{a_{0}+a_{1}, a_{2}, \ldots}^{-1} \otimes \Theta^{p}(\mathcal{L})_{a_{0}, a_{1}+a_{2}, \ldots} \otimes \Theta^{p}(\mathcal{L})_{a_{0}, a_{1}, \ldots}^{-1} \cong 1$

With these observations, one makes the following definition:

3.4 Definition Let $\mathcal{L}$ be a line bundle over a group $G$. A $\Theta^{p}$-structure on $\mathcal{L}$ is a trivialization $s$ of the line bundle $\Theta^{p}(\mathcal{L})$ such that

(1) (rigidity) for $p>0, s$ is a rigid section;

(2) (symmetry) for $p>0, s$ is symmetric in the sense that for each $\sigma \in \Sigma_{p}$, we have $\xi_{\sigma} \pi_{\sigma}^{*} s=s$;

(3) (cocycle condition) for $p>1$, the section

$$
s\left(a_{1}, a_{2}, \ldots\right) \otimes s\left(a_{0}+a_{1}, a_{2}, \ldots\right)^{-1} \otimes s\left(a_{0}, a_{1}+a_{2}, \ldots\right) \otimes s\left(a_{0}, a_{1}, \ldots\right)^{-1}
$$

corresponds to 1 under the isomorphism (3.3). 
For simplicity a $\Theta^{p}$-structure on $G$ will mean a $\Theta^{p}$-structure on the ideal sheaf $\mathcal{I}_{G}(0)$. A $\Theta^{3}$-structure is known as a cubical structure [9]. We write $C^{p}(G ; \mathcal{L})$ for the set of $\Theta^{p}$-structures on $\mathcal{L}$. Note that $C^{0}(G ; \mathcal{L})$ is just the set of trivializations of $\mathcal{L}$, and $C^{1}(G ; \mathcal{L})$ is the set of rigid trivializations of $\Theta^{1}(\mathcal{L})$. Suppose that $G$ begins life as a group over some base $S$. If $X$ is another object over $S$, then we can write $G_{X}$ for $G$ considered as a group over the base $X$, and so forth. In that case, we may write $C^{p}(G, X ; \mathcal{L})$ for the set of $\Theta^{p}$-structures on $\mathcal{L}$ over $G_{X}$.

Note that $\Theta^{p}$ can also be defined on sections: if $s$ is a section of $\mathcal{L}$, then $\Theta^{p} s$ is a section of $\Theta^{p} \mathcal{L}$. In particular if $s$ is a trivialization of $\mathcal{L}$, then $\Theta^{p} s$ is a $\Theta^{p}$-structure on $\mathcal{L}$.

It is important to observe that $\Theta^{p+1} \mathcal{L}$ can be constructed from $\Theta^{p} \mathcal{L}$ using the group structure in only one factor of $G^{p}$. Precisely, if $\mathcal{M}$ is a line bundle over $G \times X$, then we write $\delta \mathcal{M}$ for the line bundle over $G \times G \times X$ given by the formula

$$
\delta \mathcal{M} \stackrel{\text { def }}{=} \frac{\pi_{1}^{*} \mathcal{M} \pi_{2}^{*} \mathcal{M}}{\mu_{12}^{*} \mathcal{M} \mu_{\varnothing}^{*} \mathcal{M}} .
$$

That is,

$$
\delta \mathcal{M}_{a, b, x}=\frac{\mathcal{M}_{a, x} \mathcal{M}_{b, x}}{\mathcal{M}_{a+b, x} \mathcal{M}_{0, x}} .
$$

Let's write $G \vee G$ for the "wedge"

$$
G \vee G \stackrel{\text { def }}{=}(G \times\{0\}) \cup(\{0\} \times G) \subset G \times G .
$$

Notice that $\left.(\delta \mathcal{M})\right|_{(G \vee G) \times X}$ is canonically trivialized, and that from a section $s$ of $\mathcal{M}$ we obtain a section $\delta s$ of $\delta \mathcal{M}$ in the obvious way.

3.5 Proposition (1) For $p \geq 1$, there is a canonical isomorphism of rigid line bundles

$$
\begin{array}{ll} 
& \Theta^{p+1} \mathcal{L} \cong \delta \Theta^{p} \mathcal{L}, \\
\text { and so } & \Theta^{p+1} \mathcal{L} \cong \delta^{p} \Theta^{1} \mathcal{L} .
\end{array}
$$

(2) Using this identification, $\delta$ induces a homomorphism

$$
\delta: C^{p}\left(G^{p}, \mathcal{L}\right) \rightarrow C^{p+1}\left(G^{p+1}, \mathcal{L}\right) .
$$

(3) For $p \geq 1, \Theta^{p} \mathcal{L}$ is trivialized over the "fat wedge": if

$$
i: G^{p-1} \rightarrow G^{p}
$$


is any of the $p$ inclusions obtained using the identity of $G$, then $i^{*} \Theta^{p} \mathcal{L}$ is canonically trivialized. Moreover, if $s$ is a $\Theta^{p}$-structure, then $i^{*} s$ coincides with this trivialization.

Proof Items (1) and (2) are straightforward.

For (3), the case $p=1$ is obvious. For $p>1$, observe that by symmetry it suffices to treat the case that the identity goes to the first factor. In that case

$$
\Theta^{p} \mathcal{L}_{0, a_{2} \ldots}=\delta \Theta^{p-1} \mathcal{L}_{0, a_{2}, \ldots}
$$

is trivial. On sections we consider the case $p=2$; the general case is similar. Using the cocycle condition we have

$$
\frac{s(0,0) s(0, b)}{s(0, b) s(0, b)}=1
$$

and since $s$ is rigid $s(0,0)=1$, and so $s(0, b)=1$ as required.

\subsection{Complex orientations and $\Theta^{p}$-structures}

We recall how $\Theta^{p}$-structures arise in the study of multiplicative complex orientations. The case $p=1$ is the classical theory of MU-orientations, as in [1]. The cases $p=2,3$ (and $p=0$ ) were studied in [5].

An even periodic ring spectrum is a ring spectrum such that $\pi_{\mathrm{odd}} E=0$, and $\pi_{2} E$ contains a unit of $\pi_{*} E$. If $E$ is such a spectrum, then

$$
G_{E} \stackrel{\text { def }}{=} \operatorname{spf} E^{0} \mathbb{C} P^{\infty}
$$

is a (commutative, one-dimensional) formal group over $S_{E} \stackrel{\text { def }}{=} \operatorname{spec} \pi_{0} E$. Let $L$ denote the tautological bundle over $\mathbb{C} P^{\infty}$. The zero section

$$
\zeta: \mathbb{C} P_{+}^{\infty} \rightarrow\left(\mathbb{C} P^{\infty}\right)^{L}
$$

identifies $E^{0}\left(\left(\mathbb{C} P^{\infty}\right)^{L}\right)$ with the (global sections of the) ideal $\mathcal{I}(0)$ of functions on $G_{E}$ which vanish at the identity. The inclusion

$$
S^{2}=(*)^{L} \rightarrow\left(\mathbb{C} P^{\infty}\right)^{L}
$$

induces isomorphisms

$$
\pi_{2} E=\widetilde{E}^{0} S^{2} \cong 0^{*} \mathcal{I}(0) \cong \omega,
$$

identifying $\pi_{2} E$ with the (global sections) of the sheaf $\omega$ of invariant differentials on $G_{E}$. 
A map of ring spectra

$$
\mathrm{MU}\langle 2 p\rangle \rightarrow E
$$

gives rise to a map of spectra

$$
\left(\left(\mathbb{C} P^{\infty}\right)^{p}\right)^{\Pi\left(1-L_{i}\right)} \rightarrow \mathrm{MU}\langle 2 p\rangle \rightarrow E .
$$

The Thom isomorphism in this context can be interpreted as giving a natural isomorphism of

$$
E^{0}\left(\left(\mathbb{C} P^{\infty}\right)^{p}\right)=\mathcal{O}_{G_{E}^{p}}
$$

modules

$$
E^{0}\left(\left(\left(\mathbb{C} P^{\infty}\right)^{p}\right)^{\Pi\left(1-L_{i}\right)}\right) \cong \Gamma \Theta^{p} \mathcal{I}(0)
$$

inducing a map

$$
\operatorname{RingSpectra}(\mathrm{MU}\langle 2 k\rangle, E) \rightarrow C^{p}\left(G_{E}^{p} ; \mathcal{I}(0)\right) .
$$

About this situation there is the following result of [5].

3.7 Theorem For $0 \leq p \leq 3$ the natural map

$$
\text { RingSpectra }(\operatorname{MU}\langle 2 p\rangle, E) \rightarrow C^{p}\left(G_{E}^{p} ; \mathcal{I}(0)\right)
$$

is an isomorphism. If $s \in C^{p}\left(G_{E}^{p} ; \mathcal{I}(0)\right)$ corresponds to a map $\mathrm{MU}\langle 2 p\rangle \rightarrow E$, then the map

$$
\mathrm{MU}\langle 2 p+2\rangle \rightarrow \mathrm{MU}\langle 2 p\rangle \stackrel{s}{\rightarrow} E
$$

corresponds to $\delta s \in C^{p+1}\left(G_{E}^{p+1} ; \mathcal{I}(0)\right)$.

3.9 Example A map of ring spectra

$$
\mathrm{MU}\langle 0\rangle=\mathrm{MP} \rightarrow E
$$

corresponds to generator $x$ of $\mathcal{I}(0)$, which is to say an coordinate on $G_{E}$, or equivalently an element

$$
U \in E^{0}\left(\left(\mathbb{C} P^{\infty}\right)^{L}\right)
$$

whose image $x=\zeta^{*} U$ is a generator of $E^{0} \mathbb{C} P^{\infty}$.

3.10 Example A map of ring spectra

$$
\mathrm{MU}\langle 2\rangle=\mathrm{MU} \rightarrow E
$$

Algebraic 83 Geometric Topology, Volume 8 (2008) 
corresponds to a rigid trivialization of $\omega \otimes \mathcal{I}(0)^{-1}$, or equivalently of $\omega^{-1} \otimes \mathcal{I}(0)$. In topology this corresponds to a dotted arrow making the diagram

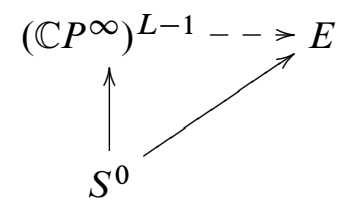

commute, which is the description of complex orientations of $E$ in [1].

Because of Theorem 3.7, if

$$
s: \mathrm{MU}\langle 2 p\rangle \rightarrow E
$$

is an orientation, we write $\delta s$ for the induced map (3.8).

\section{Sharp}

For $p \geq 1$, a $\Theta^{p+1}$-structure on $G$ nearly defines a $\Theta^{p}$-structure in the first $p$ variables. We shall develop this idea in two ways, but, as we explain in the introduction, the main point is the following. For concreteness we let $G$ be a formal group over a ring $R$, and consider the case $p=1$. Let $\mathcal{I}=\mathcal{I}(0)$ be the ideal sheaf of functions vanishing at the origin. In punctual notation, if $a, b$ represent points of $G$ then

$$
\left(\Theta^{2} \mathcal{I}\right)_{a, b} \cong \frac{\mathcal{I}_{a}}{\mathcal{I}_{0}} \frac{\mathcal{I}_{a+b}}{\mathcal{I}_{b}} \cong\left(\Theta^{1} \mathcal{I}\right)_{a} \frac{\mathcal{I}_{a+b}}{\mathcal{I}_{b}} .
$$

In pullback notation,

$$
\Theta^{2} \mathcal{I} \cong \pi_{1}^{*}\left(\Theta^{1} \mathcal{I}\right) \cdot \frac{\mu^{*} \mathcal{I}}{\pi_{2}^{*} \mathcal{I}} .
$$

Now suppose that we have a coordinate on $G$. Let

$$
F(x, y)=x+y+O(x y)
$$

be the resulting formal group law, so

$$
\mathcal{O}_{G \times G} \cong R[[x, y]]
$$

with respect to which

$$
\mu^{*} \mathcal{I}=(F(x, y))
$$

4.1 Lemma $F(x, y)$ is a unit of $R((y))[[x]]$, and so under the ring homomorphism

$$
f: R[[x, y]] \rightarrow S=R((y))[[x]],
$$


we have

$$
f^{*} \mu^{*} \mathcal{I}=S .
$$

That is, the ideal sheaf $\mu^{*} \mathcal{I}$ becomes trivial after pulling back along

$$
\operatorname{spec}(R((y))[[x]]) \rightarrow \operatorname{spec} \mathcal{O}_{G \times G},
$$

and so also over

$$
\operatorname{spf}(R((y))[[x]])
$$

Similar remarks hold for the ideal $\pi_{2}^{*} \mathcal{I}$.

Proof If we expand $F$ as a power series in $x$, with coefficients power series in $y$,

$$
F(x, y)=y+\sum_{i \geq 1} a_{i}(y) x^{i}
$$

then the constant term $y$ is a unit of $R((y))$.

In order to take advantage of this observation systematically, we introduce the following variant of $\Theta^{p}$. For $p \geq 0$, let $\Theta_{*}^{p} \mathcal{L}$ be the line bundle over $G^{p+1}$ given by the formula

$$
\Theta_{*}^{p} \mathcal{L} \stackrel{\text { def }}{=} \bigotimes_{I \subseteq\{1, \ldots, p\}}\left(\mu_{I \cup\{p+1\}}^{*} \mathcal{L}\right)^{(-1)^{|I|+1}}
$$

4.3 Example In punctual notation,

$$
\begin{aligned}
\left(\Theta_{*}^{1} \mathcal{L}\right)_{a, b} & =\frac{\mathcal{L}_{a+b}}{\mathcal{L}_{b}} \\
\left(\Theta_{*}^{2} \mathcal{L}\right)_{a, b, c} & =\frac{\mathcal{L}_{a+c} \mathcal{L}_{b+c}}{\mathcal{L}_{c} \mathcal{L}_{a+b+c}} .
\end{aligned}
$$

The important relationships between $\Theta^{p}$ and $\Theta_{*}^{p}$ are given by the following result; recall that

$$
\hat{\pi}_{p+1}: G^{p+1} \rightarrow G^{p}
$$

denotes projection to the first $p$ factors.

\subsection{Proposition (1) For $p>0$,}

$$
\Theta^{p} \mathcal{L} \cong\left(\left.\Theta_{*}^{p} \mathcal{L}\right|_{G^{p} \times 0}\right)^{-1} .
$$

(2) (Pascal's Triangle)

$$
\Theta^{p+1} \mathcal{L} \cong \hat{\pi}_{p+1}^{*} \Theta^{p} \mathcal{L} \otimes \Theta_{*}^{p} \mathcal{L} .
$$


4.5 Example For example,

$$
\begin{aligned}
\left(\Theta^{2} \mathcal{L}\right)_{a, b} & =\frac{\mathcal{L}_{0}}{\mathcal{L}_{a}} \frac{\mathcal{L}_{a+b}}{\mathcal{L}_{b}} \cong\left(\Theta^{1} \mathcal{L}\right)_{a} \otimes\left(\Theta_{*}^{1} \mathcal{L}\right)_{a, b} \\
\left(\Theta^{3} \mathcal{L}\right)_{a, b, c} & =\frac{\mathcal{L}_{0} \mathcal{L}_{a+b}}{\mathcal{L}_{a} \mathcal{L}_{b}} \frac{\mathcal{L}_{a+c} \mathcal{L}_{b+c}}{\mathcal{L}_{c} \mathcal{L}_{a+b+c}} \cong\left(\Theta^{2} \mathcal{L}\right)_{a, b} \otimes\left(\Theta_{*}^{2} \mathcal{L}\right)_{a, b, c} .
\end{aligned}
$$

We are guided by the idea that a section of $\Theta_{*}^{2} \mathcal{I}(0)$ restricts to a holomorphic function on the subspace $X \subseteq G^{3}$ where $a$ and $b$ are small compared to $c$. After all, the divisor of $\Theta_{*}^{2} \mathcal{I}(0)$ is

$$
[a+c=0]+[b+c=0]-[c=0]-[a+b+c=0],
$$

and this divisor intersects $X$ trivially. In the case of an elliptic curve $C$ over $S$, we can take

$$
X=\widehat{C}^{2} \times(C \backslash 0) .
$$

By this we mean the formal scheme over $U=(C \backslash 0)$ which is the pullback of $\widehat{C}^{2}$ in the diagram

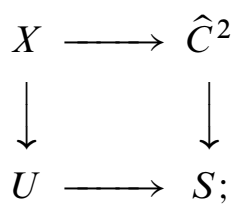

in particular $\mathcal{O}_{X}$ is the completed tensor product

$$
\mathcal{O}_{X}=\mathcal{O}_{\widehat{C}^{2}} \hat{\otimes}_{\mathcal{O}_{S}} \mathcal{O}_{U}
$$

The case of a formal group $G$ over $S=\operatorname{spec} R$ is trickier. If $y$ is a coordinate on $G$, then

$$
\mathcal{O}_{G} \cong R[[y]]
$$

with respect to which

$$
\mathcal{I}(0)=(y) .
$$

One candidate for $G \backslash 0$ is then "spf $R((y))$," but if $T$ is a discrete $R$-algebra, then the set of continuous maps $R((y))$ to $T$ is the empty set of nilpotent units in $T$.

Let

$$
R_{1}=\underset{k}{\operatorname{colim}} \mathcal{I}(0)^{-k} \mathcal{O}_{G} \cong R((y)),
$$

and let $U_{1}=\operatorname{spec} R_{1}$. Let $G_{1}$ and $G_{\circ}$ be formal groups obtained by pulling back $G$ in the diagram

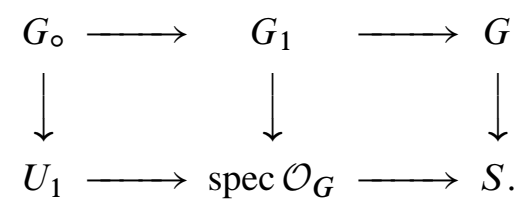


By this we mean explicitly that

$$
\mathcal{O}_{G_{\circ}} \cong R((y))[[x]] .
$$

The subscript 1 on indicates that $G_{1}$ lives over a base with one power series variable. The subscript 1 on $U_{1}$ indicates that $\mathcal{O}_{U_{1}}$ has one Laurent series variable, coming from "removing the identity section" in $\operatorname{spec} \mathcal{O}_{G}$. The subscript $\circ$ indicates that $G_{\circ}$ lives over a base with a hole in it.

We write $\mathcal{I}$ for the ideal sheaf $\mathcal{I}(0)$. If it is necessary to distinguish between $\mathcal{I}_{G}(0)$ and $\mathcal{I}_{G_{\circ}}(0)$, then we write $\mathcal{I}_{\circ}$ for the latter.

The argument of Lemma 4.1 shows that $\Theta_{*}^{p} \mathcal{I}$ becomes trivial over $G_{\circ}^{p}$, and so a section of $\Theta^{p+1} \mathcal{I} \cong \hat{\pi}_{p+1}^{*} \Theta^{p} \mathcal{I} \otimes \Theta_{*}^{p} \mathcal{I}$ gives rise to a section of $\Theta^{p} \mathcal{I}_{\circ}$.

4.7 Definition If $s$ is a section $\Theta^{p+1} \mathcal{I}$, we write $s^{\sharp}$ for resulting section of $\Theta^{p} \mathcal{I}_{0}$.

4.8 Proposition If $s$ is a $\Theta^{p+1}$-structure, then $s^{\sharp}$ is a $\Theta^{p}$-structure, and so we have a homomorphism

$$
C^{p+1}(G ; \mathcal{I}) \rightarrow C^{p}\left(G_{\circ} ; \mathcal{I}\right)
$$

Proof We need to check the rigidity, symmetry, and cocycle conditions. Let $s$ be a $\Theta^{p}$-structure on $\mathcal{I}$.

The zero section of $G_{\circ}$ is induced by the map

$$
\begin{aligned}
G & \rightarrow G_{1}^{p} \cong G^{p+1} \\
c & \mapsto(0, \ldots, 0, c) .
\end{aligned}
$$

We showed in Proposition 3.5 that

$$
s(0, \ldots, 0, c)=1 .
$$

The symmetry condition for $s^{\sharp}$ follows easily from the symmetry condition for $s$.

For $p=1$ the cocycle condition is empty. For $p \geq 2$, the cocycle condition for $s$ does not involve the last variable and thus gives the cocycle condition for $s^{\#}$.

4.9 Example If $s \in C^{2}(G ; \mathcal{I})$, then $s^{\sharp} \in C^{1}\left(G_{\circ} ; \mathcal{I}\right)$. Note that then

$$
\delta\left(s^{\sharp}\right) \in C^{2}\left(G_{\circ} ; \mathcal{I}\right),
$$

and so we have a homomorphism

$$
C^{2}(G ; \mathcal{I}) \rightarrow C^{2}\left(G_{\circ} ; \mathcal{I}\right)
$$


4.11 Example If $t \in C^{3}(G ; \mathcal{I})$, then $t^{\#} \in C^{2}\left(G_{\circ} ; \mathcal{I}\right)$. In particular, if $s \in C^{2}(G ; \mathcal{I})$ then

$$
(\delta s)^{\sharp} \in C^{2}\left(G_{\circ} ; \mathcal{I}\right),
$$

and so again we have a homomorphism

$$
C^{2}(G ; \mathcal{I}) \rightarrow C^{2}\left(G_{\circ} ; \mathcal{I}\right)
$$

4.13 Proposition The homomorphisms (4.10) and (4.12) coincide: for $s \in C^{2}(G ; \mathcal{I})$ we have

$$
\delta\left(s^{\#}\right)=(\delta s)^{\#}
$$

in $C^{2}\left(G_{\circ} ; \mathcal{I}\right)$.

Proof The formula for $\delta s$ involves only the first variable of $s$, while the construction of $s^{\#}$ involves only the last.

\section{$4.14 \Theta^{l}$-structures of $\Theta^{k}$-structures}

We describe another approach to the sharp construction which was the starting point our investigation.

If $G$ is a formal group over $S$, then we can regard $G^{l}$ as a group in the first variable, over the base $G^{l-1}$. In general, we write $G_{l-1}^{k}$ for the pullback

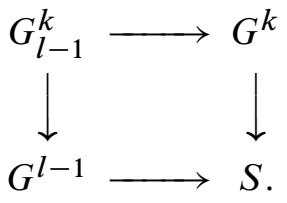

Of course we have

$$
G_{l-1}^{k} \cong G^{k+l-1},
$$

and we shall consider the last $l-1$ factors to be the "base."

If $\mathcal{L}$ is a line bundle over $G$, then we can consider $\Theta^{l} \mathcal{L}$ as a line bundle $G_{l-1}$. We then have two line bundles over $G_{l-1}^{k}$, namely $\Theta^{k}\left(\Theta^{l}(\mathcal{L})\right)$ and $\Theta^{k+l-1}(\mathcal{L})$. Explicitly,

$$
\Theta^{k}\left(\Theta^{l}(\mathcal{L})\right)=\bigotimes_{I \subseteq\{1, \ldots, k\}} \mu_{I}^{*}\left(\Theta^{l}(\mathcal{L})\right)^{(-1)^{|I|}},
$$

where $\quad \mu_{I}: G_{l-1}^{k} \cong G^{k+l-1} \rightarrow G^{l} \cong G_{l-1}$

is the map $\mu_{I}$ of Section 2 on the first $k$ factors of $G^{k+l-1}$, and the identity on the last $l-1$. 
4.16 Proposition For $k \geq 1$ and $l \geq 2$, there is a canonical isomorphism

$$
\zeta_{l, k}: \Theta^{k+l-1}(\mathcal{L})^{-1} \cong \Theta^{k}\left(\Theta^{l}(\mathcal{L})\right)
$$

of line bundles over $G^{k+l-1}$. In particular

$$
\Theta^{k+1}(\mathcal{L})^{-1} \cong \Theta^{k}\left(\Theta^{2}(\mathcal{L})\right)
$$

Proof Represent $I \subset\{1, \ldots, k\}$ and $J \subset\{1, \ldots, l\}$ by vectors $\left(i_{1}, \ldots, i_{k}\right) \in \mathbb{F}_{2}^{k}$ and $\left(j_{1}, \ldots, j_{l}\right) \in \mathbb{F}_{2}^{l}$ respectively. Then

$$
\mu_{J} \circ \mu_{I}=\mu_{J \circ I}
$$

where

$$
J \circ I \subset\{1, \ldots, k+l-1\}
$$

denotes the subset represented by

Now

$$
\begin{gathered}
\left(i_{1} j_{1}, \ldots, i_{k} j_{1}, j_{2}, \ldots, j_{l}\right) \in \mathbb{F}_{2}^{k+l-1} . \\
\Theta^{k} \Theta^{l}(\mathcal{L})=\bigotimes_{I} \mu_{I}^{*}\left(\bigotimes_{J} \mu_{J}^{*}(\mathcal{L})^{(-1)^{|J|+1}}\right)^{(-1)^{|I|}} \\
\cong \bigotimes_{I} \bigotimes_{1 \in J} \mu_{J \circ I}^{*}(\mathcal{L})^{(-1)^{|I|+|J|+1}}
\end{gathered}
$$

because the factors coming from terms with $1 \notin J$ all appear equally often with their inverse and therefore cancel out.

4.17 Example We will mainly be interested in the case $k=l=2$. At a point $(a, b, c)$ of $G_{1}^{2} \cong G^{3}$, we have

$$
\begin{aligned}
\Theta^{2}\left(\Theta^{2} \mathcal{L}\right)_{a, b, c} & \cong \frac{\left(\Theta^{2} \mathcal{L}\right)_{a+b, c}\left(\Theta^{2} \mathcal{L}\right)_{0, c}}{\left(\Theta^{2} \mathcal{L}\right)_{a, c}\left(\Theta^{2} \mathcal{L}\right)_{b, c}} \\
& \cong \frac{\mathcal{L}_{a+b+c} \mathcal{L}_{a} \mathcal{L}_{b} \mathcal{L}_{c}}{\mathcal{L}_{0} \mathcal{L}_{a+b} \mathcal{L}_{a+c} \mathcal{L}_{b+c}} \\
& \cong\left(\Theta^{3} \mathcal{L}\right)^{-1}
\end{aligned}
$$

Along the same lines, we note that

$$
\Theta^{2}\left(\Theta^{2} \mathcal{L}\right) \cong \Theta^{2}\left(\frac{\mu^{*} \mathcal{L}}{\pi_{1}^{*} \mathcal{L}}\right),
$$

and in general

$$
\Theta^{p}\left(\Theta^{2} \mathcal{L}\right) \cong \Theta^{p}\left(\frac{\mu^{*} \mathcal{L}}{\pi_{1}^{*} \mathcal{L}}\right)
$$


4.19 Definition For a $\Theta^{k+1}$-structure $s$ on $\mathcal{L}$, let $s^{\mathrm{b}}$ be the section

$$
s^{b}:=\zeta_{2, k} s^{-1}
$$

of $\Theta^{k}\left(\Theta^{2}(\mathcal{L})\right)$.

Now we can proceed as before. The proof of Proposition 4.8 applies to give the following.

4.20 Proposition If $s$ is a $\Theta^{k+1}$-structure on $\mathcal{L}$, then $s^{b}$ is a $\Theta^{k}$-structure on $\Theta^{2}(\mathcal{L})$.

A $\Theta^{p+1}$-structure

$$
s \in C^{p+1}(G ; \mathcal{I}(0))
$$

gives rise to a $\Theta^{p}$-structure on $\Theta^{2} \mathcal{I}(0)$, and indeed using the isomorphism (4.18), an element

$$
s^{b} \in C^{p}\left(G_{1} ; \mu^{*} \mathcal{I}(0) / \pi_{1}^{*} \mathcal{I}(0)\right) .
$$

After pulling back along

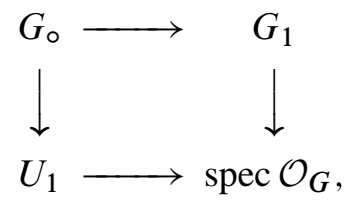

we find that the ideal $\mu^{*} \mathcal{I}(0)$ becomes trivial, and so $s^{\#}=\left(s^{b}\right)^{-1}$ can be considered to be an element

$$
s^{\#} \in C^{p}\left(G_{\circ} ; \mathcal{I}(0)\right) \text {. }
$$

\section{Application to complex orientations}

In this section we apply the results of Section 4 to orientations of ring spectra. We begin by describing the topological counterpart of the base change to $U_{1}$.

\subsection{The pro-spectrum $\mathbb{C P}_{-\infty}^{\infty}$}

We recall that just as

$$
\mathcal{O}_{G_{E}}=E^{0} \mathbb{C} P^{\infty}=\pi_{0} E^{\mathbb{C} P_{+}^{\infty}},
$$

the ring $\mathcal{O}_{U_{1}}$ arises as the cohomology of Mahowald's pro-spectrum

$$
\mathcal{O}_{U_{1}}=E^{0} \mathbb{C} P_{-\infty}^{\infty}=\pi_{0} E^{\mathbb{C} P_{-\infty}^{\infty}}
$$

and so $E \mathcal{O}_{U_{1}}=E^{\mathbb{C} P_{-\infty}^{\infty}}$. 
In more detail, if $L$ denotes the tautological line bundle over $\mathbb{C} P^{\infty}$, then $\mathbb{C} P_{-k}^{\infty}$ is the Thom spectrum of $-k L$, and

$$
\mathbb{C} P_{-\infty}^{\infty} \stackrel{\text { def }}{=}\left(\cdots \rightarrow \mathbb{C} P_{-k}^{\infty} \rightarrow \mathbb{C} P_{-k+1}^{\infty} \cdots \rightarrow \mathbb{C} P_{-1}^{\infty} \rightarrow \mathbb{C} P_{+}^{\infty}\right) .
$$

If $t$ is a coordinate on $G$, so $E^{0} \mathbb{C} P^{\infty}=E^{0}[[t]]$, then

$$
E^{0} \mathbb{C} P_{-k}^{\infty}=t^{-k} E^{0} \mathbb{C} P^{\infty},
$$

and if we define

$$
E^{\mathbb{C} P_{-\infty}^{\infty}} \stackrel{\text { def }}{=} \operatorname{hocolim}\left(E^{\mathbb{C} P_{+}^{\infty}} \rightarrow E^{\mathbb{C} P_{-1}^{\infty}} \rightarrow \cdots\right),
$$

then

$$
\pi_{0} E^{\mathbb{C} P_{-\infty}^{\infty}} \cong E^{0}((t)) .
$$

It turns out that $E^{\mathbb{C P}}-_{-\infty}^{\infty}$ is a ring spectrum, in such a way that this is an isomorphism of rings. Indeed, the ring structure is represented by a pro-diagonal. More precisely, we have the following.

\subsection{Proposition There are compatible counit maps}

$$
\mathbb{C} P_{-k}^{\infty} \rightarrow S^{0}
$$

and diagonal maps

$$
\mathbb{C} P_{-k-l}^{\infty} \rightarrow \mathbb{C} P_{-k}^{\infty} \wedge \mathbb{C} P_{-l}^{\infty},
$$

such that the obvious coassociativity, cocommutativity and counit diagrams commute, giving $\mathbb{C} P_{-\infty}^{\infty}$ the structure of a comonoid pro-spectrum.

Proof The counit map is given by

$$
\mathbb{C} P_{-k}^{\infty} \rightarrow \mathbb{C} P_{+}^{\infty} \rightarrow S^{0}
$$

which is just the map of Thom spaces associated to the map of bundles:

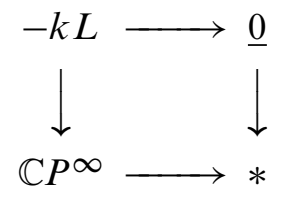

The diagonal is the map of Thom spectra associated to

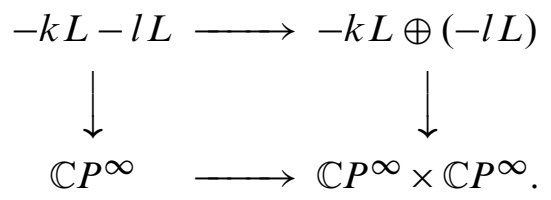

Algebraic $8 \mathcal{G}$ Geometric Topology, Volume 8 (2008) 
Using the ring spectrum structure on $E$, we have compatible maps

$$
E^{\mathbb{C} P_{-k}^{\infty}} \wedge E^{\mathbb{C} P_{-l}^{\infty}} \rightarrow E^{\mathbb{C} P_{-k}^{\infty} \wedge \mathbb{C} P_{-l}^{\infty}} \rightarrow E^{\mathbb{C} P_{-k-l}^{\infty},}
$$

and passing to colimits we have the following.

5.3 Corollary If $E$ is an even periodic ring spectrum, then $E^{\mathbb{C} P_{-\infty}^{\infty}}$ is a ring spectrum. If $G=\operatorname{spf} E^{0} \mathbb{C} P^{\infty}$ is the formal group associated to $E$, then

$$
\pi_{0} E^{\mathbb{C P}}{ }_{-\infty}^{\infty} \cong \mathcal{O}_{U_{1}},
$$

and the formal group associated to $E^{\mathbb{C} P} P_{-\infty}^{\infty}$ is $G_{\circ}$. A coordinate on $G$ gives an element $y \in \pi_{0} E^{\mathbb{C} P_{-\infty}^{\infty}}$ and $x \in E^{0}\left(\mathbb{C} P^{\infty}\right)$, in terms of which

and

$$
\begin{aligned}
\pi_{0} E^{\mathbb{C} P_{-\infty}^{\infty}} & \cong E^{0}((y)) \\
\left(E^{\mathbb{C} P_{-\infty}^{\infty}}\right)^{0}\left(\mathbb{C} P^{\infty}\right) & \cong E^{0}((y))[[x]] \\
\left(E^{\mathbb{C} P_{-\infty}^{\infty}}\right)^{*}(X) & \cong E^{*}\left(X ; E^{*}((y))\right) .
\end{aligned}
$$

More generally,

5.4 Remark The fact that $E^{\mathbb{C} P{ }_{-\infty}^{\infty}}$ is a ring spectrum is well-known. For example, this ring structure was studied by Cohen, Jones and Segal in [10]. Also, as we learned from Hal Sadofsky, $E^{\mathbb{C} P_{-\infty}^{\infty}}$ is the $\mathbb{T}$-fixed point spectrum of the Tate spectrum $t_{\mathbb{T}}(E)$ of $E$, considered as a $\mathbb{T}$-spectrum with the trivial action. As such, it follows from work of Greenlees and May, for example Proposition 3.5 of [19], that $E^{\mathbb{C} P_{-\infty}^{\infty}}$ is a ring spectrum. Indeed, McClure shows in [30] that if $E$ is an $E_{\infty}$ ring spectrum, then so is $E^{\mathbb{C} P_{-\infty}^{\infty}} \cong\left(t_{\mathbb{T}}(E)\right)^{\mathbb{T}}$.

\subsection{New orientations from old}

Let $E$ be an even periodic ring spectrum with formal group $G=G_{E}$. Suppose that we are given a map of ring spectra

$$
\mathrm{MU}\langle 2 p\rangle \rightarrow E
$$

and so a $\Theta^{p}$-structure

$$
s \in C^{p}(G ; \mathcal{I}(0)) .
$$

Proposition 4.8 gives the section

$$
s^{\#} \in C^{p-1}\left(G_{\circ} ; \mathcal{I}(0)\right) .
$$

Theorem 3.7 and Corollary 5.3 imply that in terms of orientations we have the following result. 
5.6 Proposition If $p \leq 4$, then the function

$$
(-)^{\sharp}: C^{p}(G ; \mathcal{I}) \rightarrow C^{p-1}\left(G_{\circ} ; \mathcal{I}\right)
$$

determines a function

$$
\operatorname{RingSpectra}(\mathrm{MU}\langle 2 p\rangle, E) \rightarrow \operatorname{RingSpectra}\left(\operatorname{MU}\langle 2(p-1)\rangle, E^{\mathbb{C P}{ }_{-\infty}^{\infty}}\right) .
$$

5.7 Example Thus an SU-orientation of a complex-orientable spectrum $E$ gives rise to a complex orientation of $E^{\mathbb{C} P_{-}^{\infty}}$. We thank the referee for pointing out to us that this natural transformation arises from a canonical map of ring spectra

$$
\mathrm{MU} \rightarrow \mathrm{MSU}^{\mathbb{C} P} \infty_{-\infty}^{\infty}
$$

Indeed, Adam's theory of complex orientations (described in Example 3.10) says that to give a map of ring spectra (5.8) is equivalent to giving a map

$$
f:\left(\mathbb{C} P^{\infty}\right)^{1-L} \rightarrow \operatorname{MSU}^{\mathbb{C} P_{-\infty}^{\infty}}
$$

making the diagram

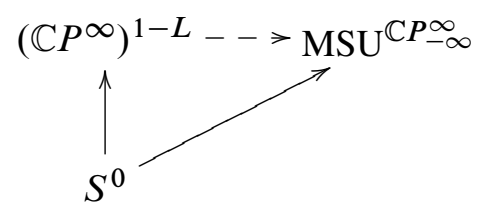

commute. Our map arises from a compatible family of maps

$$
f_{k}:\left(\mathbb{C} P^{\infty}\right)^{1-L} \wedge \mathbb{C} P_{-k}^{\infty} \cong\left(\mathbb{C} P^{\infty} \times \mathbb{C} P^{\infty}\right)^{1-L-k M} \rightarrow \mathrm{MSU},
$$

where we have written $M$ for the tautological bundle over the second factor $\mathbb{C} P^{\infty}$. To give such a map, note that $(1-L)(1-M)$ is an SU-bundle, and so we have a map

$$
\left(\mathbb{C} P^{\infty} \times \mathbb{C} P^{\infty}\right)^{(1-L)(1-M)} \rightarrow \mathrm{MSU} .
$$

Moreover for $k \geq 1$ the difference

$$
(1-L)(1-M)-(1-L-k M)=L M+(k-1) M
$$

is a genuine vector bundle, and so we have the relative zero section $\zeta$ in the sequence

$$
\begin{aligned}
f_{k}:\left(\mathbb{C} P^{\infty}\right)^{(1-L)} \wedge \mathbb{C} P_{-k}^{\infty} \cong\left(\mathbb{C} P^{\infty} \times \mathbb{C} P^{\infty}\right)^{1-L-k M} & \\
& \stackrel{\zeta}{\rightarrow}\left(\mathbb{C} P^{\infty} \times \mathbb{C} P^{\infty}\right)^{(1-L)(1-M)} \rightarrow \mathrm{MSU} .
\end{aligned}
$$


5.9 Example Similarly we can describe the map

$$
\mathrm{MSU} \rightarrow \mathrm{MU}\langle 6\rangle^{\mathbb{C P}} P_{-\infty}^{\infty}
$$

which corresponds to the natural transformation in the Proposition. Let $V_{k}$ denote the tautological bundle over $\operatorname{BSU}(k)$. The bundle $\left(k-V_{k}\right)(1-M)$ over $\operatorname{BSU}(k) \times \mathbb{C} P^{\infty}$ is classified by a map

$$
\mathrm{BSU}(k) \times \mathbb{C} P^{\infty} \rightarrow \mathrm{BU}\langle 6\rangle .
$$

The difference

$$
\left(k-V_{k}\right)(1-M)-\left(k-V_{k}-k M\right)=(k-1) M+V M
$$

is a genuine bundle for $k \geq 1$, and so we have a map of Thom spectra

$$
(\operatorname{BSU}(k))^{\left(k-V_{k}\right)} \wedge \mathbb{C} P_{-k}^{\infty} \rightarrow\left(\operatorname{BSU}(k) \times \mathbb{C} P^{\infty}\right)^{\left(k-V_{k}\right)(1-M)} \rightarrow \operatorname{MU}\langle 6\rangle .
$$

Taking adjoints and passing to colimits gives the desired map.

\subsection{Two-variable genera}

Suppose that $E$ is an even periodic ring spectrum, and let $G=G_{E}$. Let

$$
i: S^{2} \rightarrow \mathbb{C} P^{\infty}
$$

denote the inclusion of the bottom cell. Then

$$
E^{0} S^{2}=\pi_{2} E \cong \mathcal{I} / \mathcal{I}^{2}
$$

is the dual Lie algebra of $G$, and if $f \in E^{0} \mathbb{C} P^{\infty}$ is considered as a function on $G$, then

$$
i^{*} f=d f_{0} .
$$

If $d f_{0}$ is a generator of $\pi_{2} E$, then by Theorem 3.7, $f$ determines a map of ring spectra

$$
\mathrm{MP}=\mathrm{MU}\langle 0\rangle \rightarrow E,
$$

and so a complex orientation

$$
\mathrm{MU}=\mathrm{MU}\langle 2\rangle \rightarrow E,
$$

for which the corresponding element of $C^{1}(G ; \mathcal{I}(0))$ is

$$
s=\frac{d f_{0}}{f} .
$$

Again by Theorem 3.7, the SU-orientation

$$
\mathrm{MSU}=\mathrm{MU}\langle 4\rangle \rightarrow \mathrm{MU} \rightarrow E
$$

Algebraic 83 Geometric Topology, Volume 8 (2008) 
corresponds to the element

$$
\delta s=\frac{d f_{0} \mu^{*} f}{\pi_{1}^{*} f \pi_{2}^{*} f}
$$

of $C^{2}(G ; \mathcal{I}(0))$.

Now Proposition 4.8 implies that

$$
(\delta s)^{\sharp} \in C^{1}\left(G_{\circ} ; \mathcal{I}(0)\right),
$$

and so determines a complex orientation

$$
\mathrm{MU} \rightarrow E^{\mathbb{C} P_{-\infty}^{\infty}}
$$

5.12 Definition Let $E$ be an even-periodic, homotopy-commutative ring spectrum, and let

$$
\varphi: \mathrm{MU} \rightarrow E
$$

be a multiplicative complex orientation, associated to a $\Theta^{1}$-structure $s \in C^{1}\left(G_{E} ; \mathcal{I}(0)\right)$. The adjoint genus of $\varphi$ is the map of ring spectra

$$
\widehat{\varphi}: \mathrm{MU} \rightarrow E^{\mathbb{C P}-\infty}
$$

associated to the element $(\delta s)^{\sharp} \in C^{1}\left(G_{\circ} ; \mathcal{I}(0)\right)$.

It is illuminating to spell this out in terms of a coordinate $t$ on $G$, so that $E^{0} \mathbb{C} P^{\infty} \cong$ $E^{0}\left[[t]\right.$. Then $\mathcal{O}_{G \times G}=\pi_{0} E\left[\left[t_{1}, t_{2}\right]\right]$, and the group structure of $G$ can be expressed as a formal group law

$$
\mu^{*} t=F\left(t_{1}, t_{2}\right)
$$

We can write

$$
f=f(t) \in E^{0}[[t]]
$$

with $f^{\prime}(0) \in\left(\pi_{0} E\right)^{\times}$, and then $\delta f$ is given by the expression

$$
\delta f\left(t_{1}, t_{2}\right)=\frac{d f_{0} f\left(F\left(t_{1}, t_{2}\right)\right)}{f\left(t_{1}\right) f\left(t_{2}\right)} .
$$

The adjoint genus is the genus associated to this expression, with $t_{1}$ considered as the coordinate on the group, and $t_{2}$ considered as an element of $\pi_{0} E^{\mathbb{C} P{ }_{-\infty}^{\infty}} \cong \pi_{0} E\left(\left(t_{2}\right)\right)$.

5.13 Example If $E$ is rational, then we can choose the coordinate $t$ so that $F$ is the additive group. Set $x=t_{1}$ and $z=t_{2}$ above, and let

$$
g(x, z)=\frac{f(x+z)}{f(x) f(z)} .
$$

Algebraic 83 Geometric Topology, Volume 8 (2008) 
If $M$ is a complex manifold with total Chern class

$$
c(M)=\prod\left(1+x_{i}\right)
$$

then by the topological Riemann-Roch theorem (see for example Dyer [14] and Rudyak [32]), the adjoint genus of $M$ associated to $f$ is

$$
\int_{M} \prod_{i} \frac{x_{i}}{g\left(x_{i}, z\right)}
$$

\section{The sigma function and the two-variable elliptic genus}

We mentioned in the introduction that the Tate elliptic curve $C_{\text {Tate }}$ over $\mathbb{Z}[[q]]$ gives rise to an elliptic spectrum $K_{\text {Tate }}$, whose underlying spectrum is $K[[q]]$. In this section we show that when the analysis in Section 5.10 is applied to the complex orientation of $K_{\text {Tate }}$ given by the Weierstrass sigma function, the resulting two-variable genus is the two-variable elliptic genus.

\subsection{The sigma function and $\Phi$}

Let $\Phi$ and $\sigma$ be the power series

$$
\begin{aligned}
\Phi(u, q) & =\left(1-u^{-1}\right) \prod_{n \geq 1} \frac{\left(1-q^{n} u\right)\left(1-q^{n} u^{-1}\right)}{\left(1-q^{n}\right)^{2}} \\
\sigma(u, q) & =u^{1 / 2} \Phi(u, q) \\
& =\left(u^{1 / 2}-u^{-1 / 2}\right) \prod_{n \geq 1} \frac{\left(1-q^{n} u\right)\left(1-q^{n} u^{-1}\right)}{\left(1-q^{n}\right)^{2}} .
\end{aligned}
$$

By considering $u$ to be a complex line bundle, one sees that they define the same genus

$$
\mathrm{MSU} \rightarrow K[[q]] .
$$

The genus associated to $\Phi$ factors through MU, while the genus associated to $\sigma$ factors through MSpin; as such it is known as the Witten genus [22].

We may view $\Phi$ and $\sigma$ as functions of variables $x$ and $\tau$ by setting

$$
\begin{aligned}
u^{r} & =e^{r x} \\
q & =e^{2 \pi i \tau}
\end{aligned}
$$

for $r \in \mathbb{Q}$. They are variants of the Weierstrass sigma function. A number of the following remarks apply to both $\sigma$ and $\Phi$, but for definiteness we focus on $\Phi$. 
For $\tau \in \mathfrak{h}, 0<|q|<1$, and so $\Phi$ is a holomorphic function of $(x, \tau) \in \mathbb{C} \times \mathfrak{h}$. For fixed $\tau, \Phi(x, \tau)$ vanishes to first order when $x$ is a point of the lattice

$$
\Lambda=2 \pi i \mathbb{Z}+2 \pi i \tau \mathbb{Z},
$$

and has no other zeroes. It is not invariant under translation by $\Lambda$ in $x$; instead we have

$$
\Phi\left(u q^{n}\right)=(-1)^{n} u^{-n} q^{-n(n+1) / 2} \Phi(u) .
$$

It follows that $\Phi$ descends to a holomorphic section of the line bundle

$$
\mathcal{A}=\frac{\mathbb{C}^{\times} \times \mathbb{C}}{(u, v) \sim\left(u q^{n}, v(-1)^{n} u^{n} q^{n(n+1) / 2}\right)}
$$

over

$$
C=\mathbb{C}^{\times} / q^{\mathbb{Z}} \cong \mathbb{C} / \Lambda,
$$

vanishing to first order at the origin. As such $\Phi$ is a trivialization of $\mathcal{A} \otimes \mathcal{I}(0)$, which is to say an isomorphism of line bundles over $C$

$$
\mathcal{A} \cong \mathcal{I}(0)^{-1} \text {. }
$$

\subsection{The adjoint of the Witten genus is the two-variable elliptic genus}

For the moment let's write $p$ for the projection

$$
p: \mathbb{C} \rightarrow C .
$$

The classical story of the sigma function implies that if $y$ and $z$ are two points of $\mathbb{C}$,

$$
W(x, y, z)=\frac{\Phi(x+y) \Phi(x+z)}{\Phi(x) \Phi(x+y+z)}
$$

considered as a function of $x$, descends to a meromorphic function on $C$ with divisor

$$
[-p(y)]+[-p(z)]-[0]-[-p(y)-p(z)] .
$$

As a function of $x$ we have

$$
\Phi(x, \tau)=x+O\left(x^{2}\right),
$$

and so via the isomorphism of formal groups

$$
\hat{p}: \widehat{\mathbb{G}}_{a}=\widehat{\mathbb{C}} \rightarrow \widehat{C},
$$

$\Phi(x, \tau)$ gives a coordinate on the formal group of $C$. As such,

$$
s=\frac{\Phi(0, \tau)}{\Phi(x, \tau)}
$$

Algebraic 83 Geometric Topology, Volume 8 (2008) 
defines an element of $C^{1}(\widehat{C}, \mathcal{I}(0))$, and so by Theorem 3.7 determines an orientation

$$
\mathrm{MU} \rightarrow H \Lambda \text {. }
$$

Here

$$
H \Lambda^{*}(X)=H P^{*}\left(X ; \mathcal{O}_{\mathfrak{h}}\right)=H^{*}\left(X ; \mathcal{O}_{\mathfrak{h}}\left[v, v^{-1}\right]\right)
$$

is periodic ordinary cohomology with coefficients in the holomorphic function on the upper half plane.

The resulting MSU orientation corresponds by Theorem 3.7 to the $\Theta^{2}$-structure

$$
\delta s=\frac{\Phi(0, \tau) \Phi(x+z, \tau)}{\Phi(x, \tau) \Phi(z, \tau)}=\frac{\sigma(0, \tau) \sigma(x+z, \tau)}{\sigma(x, \tau) \sigma(z, \tau)} \in C^{2}\left(\widehat{C}^{2} ; \mathcal{I}(0)\right) .
$$

As in Section 5.10, we then have the adjoint orientation

$$
\mathrm{MU} \rightarrow H \Lambda^{\mathbb{C} P} P_{-\infty}^{\infty}
$$

associated to the expression (6.4), now written as

$$
(\delta s)^{\sharp} \in C^{1}\left(\widehat{C}_{\circ} ; \mathcal{I}(0)\right) \text {. }
$$

The associated genus is often called the two-variable elliptic genus.

To compare the genus associated to $(\delta s)^{\#}$ to standard formulas for the two-variable genus, it is convenient to use the $q$-expansion formula for $\Phi$ and express our orientation in $K$-theory, as a map

$$
\phi: \mathrm{MU} \rightarrow K[[q]]((y)),
$$

where $K[[q]](X)=K(X ; \mathbb{Z}[[q]])$. Borisov and Libgober use $-z$ in (6.4) where we have used $z$, and so when passing to $K$-theory it is appropriate to set $y^{r}=e^{-r z}$ for $r \in \mathbb{Q}$. We then find that the genus of a manifold $M$ with complex tangent bundle $T$ of rank $d$ is related to the genus Ell $y$ of [6, equation (8)] by the formula

$$
\begin{aligned}
\phi(M, y, q) & =\sigma\left(y^{-1}, q\right)^{-d} y^{-\frac{d}{2}} . \\
\operatorname{Todd} & \left(M ; \bigotimes_{n \geq 1} \operatorname{Sym}_{q^{n}} T \otimes \operatorname{Sym}_{q^{n}} \bar{T} \otimes \Lambda_{-y q^{n-1}}(\bar{T}) \otimes \Lambda_{-y^{-1} q^{n}}(T)\right) \\
& =\sigma\left(y^{-1}, q\right)^{-d} \operatorname{Ell}_{y}(M) .
\end{aligned}
$$

Höhn includes the factor of $\sigma\left(y^{-1}, q\right)$ in the genus. In Lemma 2.5.1 of [23] Höhn also uses $-z$ where we have used $z$. He then sets $y=-e^{z}$, so our $y$ is his $-y$. With this understood, we find that

$$
\varphi_{\text {Höhn }}(M,-y, q)=\phi(M, y, q) \text {. }
$$




\subsection{Modularity of the two-variable genus of SU-manifolds}

Something interesting happens when we restrict the orientation $(\delta s)^{\#}$ back to MSU: this is the orientation

$$
\mathrm{MSU} \rightarrow H \Lambda^{\mathbb{C} P}{ }_{-\infty}^{\infty}
$$

associated to the section

$$
\delta(\delta s)^{\sharp}=\frac{\Phi(0, \tau) \Phi(x+y, \tau) \Phi(x+z) \Phi(y+z)}{\Phi(x, \tau) \Phi(y, \tau) \Phi(z) \Phi(x+y+z)}=(\delta s) W(z, y, x),
$$

of $\Theta^{2} \mathcal{I}(0)$ over $\widehat{C}_{\mathrm{o}}^{2}$. We make two related observations about expression (6.8).

(1) It is precisely the formula for the canonical $\Theta^{3}$-structure on $\mathbb{C} / \Lambda$, as explained in $[9 ; 5$, Section 2.6].

(2) Up to the indicated permutation of $x, y, z$, the factor $W$ in (6.8) is same as the $W$ in (6.3). Thus $\delta^{2} s^{\#}$ gives in fact a section of

$$
\Theta^{2} \mathcal{I}(0) \otimes \mathcal{K}_{\Lambda}
$$

where $\mathcal{K}_{\Lambda}$ denotes the meromorphic functions on $\mathbb{C} / \Lambda$. As such it determines an orientation

$$
\mathrm{MSU} \rightarrow H \mathcal{K}_{\Lambda}
$$

These observations are both complex-analytic aspects of the role of the Theorem of the Cube in the two-variable genus. In Section 7 we pursue this point of view and construct a natural genus for SU-manifolds taking values in meromorphic Jacobi forms; for the curve $\mathbb{C} / \Lambda$ over $\mathfrak{h}$ it specializes to give the two-variable genus.

\section{The Jacobi orientation}

Abel's Theorem (a particular case of the Theorem of the Cube) implies that an elliptic curve $C$ has a canonical cubical structure, that is, an element $s(C) \in C^{3}(C, \mathcal{I}(0))$. If ( $E, C, \gamma)$ is an elliptic spectrum, so $\gamma$ is an isomorphism $G_{E} \cong \widehat{C}$, then the "sigma orientation" of $(E, C, \gamma)$ is the map of ring spectra

$$
\sigma(E, C, \gamma): \operatorname{MU}\langle 6\rangle \rightarrow E
$$

associated to $\left(\gamma^{3}\right)^{*}\left(\left.s\right|_{\widehat{C}^{3}}\right)$ by Theorem 3.7 and [5].

The sigma orientation is modular in the following sense. A map of elliptic spectra

$$
(f, \alpha):(E, C, \gamma) \rightarrow\left(E^{\prime}, C^{\prime}, \gamma^{\prime}\right)
$$


is a map of ring spectra

$$
f: E \rightarrow E^{\prime}
$$

together with an isomorphism of elliptic curves

$$
\alpha: C^{\prime} \cong\left(\operatorname{spec} \pi_{0} f\right)^{*} C
$$

making the diagram

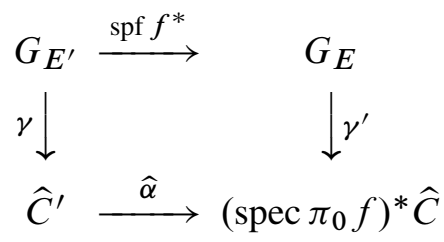

commute (recall that $\left.G_{E}=\operatorname{spf} E^{0} \mathbb{C} P^{\infty}\right)$. Given a map $(f, \alpha)$ of elliptic spectra, the following diagram commutes:

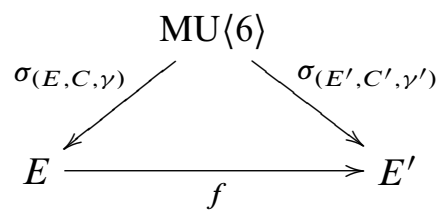

As explained in [5], the preceding discussion extends to generalized elliptic curves in the sense of [12]: if $C / S$ is a generalized elliptic curve, then its smooth locus $C^{\text {reg }}$ is a group scheme over $S$, and there is a canonical cubical structure $s(C)$ on $C^{\text {reg }}$, which restricts to a cubical structure on $\widehat{C}$, which is a one-dimensional formal group. ${ }^{3}$ The sigma orientation of the elliptic spectrum associated to the Tate curve is just the Witten genus $[22 ; 34 ; 5]$, and the modularity of the sigma orientation implies that the Witten genus of an MU $\langle 6\rangle$-manifold is the $q$-expansion of a modular form.

In this section we show that the same argument gives for any elliptic spectrum $(E, C, \gamma)$ a canonical, modular SU-orientation $J_{(E, C, \gamma)}$, taking values in the spectrum of meromorphic functions on the curve $C$. We call it the Jacobi orientation. Its value on the Tate elliptic spectrum $K_{\text {Tate }}$ is the restriction to MSU of the two-variable elliptic genus $\phi$ of (6.6), and its modularity gives a new proof of the fact that this two-variable genus is a meromorphic weak Jacobi form of index zero and weight $d$.

Let $C$ be an elliptic curve over $S=\operatorname{spec} R$, or even a generalized elliptic curve in the sense of [12]. The Jacobi orientation arises from the simple observation that in the

\footnotetext{
${ }^{3}$ In [5], a generalized elliptic curve over $S$ is defined to be a pointed $S$-scheme which is Zariski locally on $S$ isomorphic to a (possibly singular) Weierstrass curve. The analysis of [11] discussed below shows that this definition is equivalent to the definition of Deligne and Rapoport.
} 
isomorphism

$$
\Theta^{3} \mathcal{I}_{C}(0) \cong \Theta^{2} \mathcal{I}_{C}(0) \otimes \Theta_{*}^{2} \mathcal{I}_{C}(0)
$$

of line bundles over $C^{3}$, the second tensor factor is a trivial subsheaf of the meromorphic functions on the third factor $C$, by a trivialization which becomes a unit in $\mathcal{O}_{\widehat{C}^{2} \times(C \backslash 0)}$.

Let

$$
\mathcal{J}_{C} \stackrel{\text { def }}{=} \Gamma\left(\mathcal{O}_{C \backslash 0}\right),
$$

and let $\mathcal{X}_{C}=\operatorname{spec} \mathcal{J}_{C}$. Suppose for simplicity that the formal group $\widehat{C}$ admits a coordinate $t$ over $R$. Then the Riemann-Roch Theorem (for a treatment which includes generalized elliptic curves see Deligne [11]) implies that there are $x \in \Gamma\left(\mathcal{I}_{C}(0)^{-2}\right)$ and $y \in \Gamma\left(\mathcal{I}_{C}(0)^{-3}\right)$ and $a_{i} \in R$ such that

$$
\mathcal{J}_{C} \cong R[x, y] /\left(y^{2}+a_{1} x y+a_{3} y=x^{3}+a_{2} x^{2}+a_{4} x+a_{6}\right) \cong \underset{k \geq 0}{\operatorname{colim}} \Gamma\left(\mathcal{I}_{C}(0)^{-k}\right),
$$

and the natural map

$$
C \backslash 0 \rightarrow \mathcal{X}_{C}
$$

is an isomorphism. As in Section 4, let

$$
U_{1}=\operatorname{spec} \operatorname{colim}_{k} \mathcal{I}(0)^{-k} \mathcal{O}_{\widehat{C}} \cong \operatorname{spec} R((t)) .
$$

Expansion of meromorphic functions at the identity gives a ring homomorphism

$$
\mathcal{J}_{C} \rightarrow \mathcal{O}_{U_{1}}
$$

and so we have the diagram of formal groups

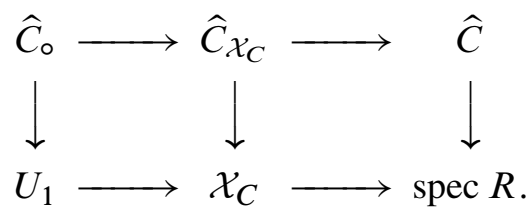

Then we have the following.

7.1 Proposition The canonical cubical structure $s(C) \in C^{3}(C, \mathcal{I}(0))$ determines a canonical and natural $\Theta^{2}$-structure

$$
s(C)^{\#} \in C^{2}\left(\widehat{C}, \mathcal{X}_{C} ; \mathcal{I}(0)\right) .
$$

This is compatible with the sharp construction on formal groups of Proposition 4.8 in the sense that

$$
\left.s(C)^{\#}\right|_{\widehat{C}_{\circ}^{2}}=\left(\left.s(C)\right|_{\widehat{C}^{3}}\right)^{\#} \in C^{2}\left(\widehat{C}_{\circ} ; \mathcal{I}(0)\right) \text {. }
$$


If $(E, C, \gamma)$ is an elliptic spectrum, then $\widehat{C}$ admits a coordinate since

$$
\widehat{C} \cong \operatorname{spf} E^{0} \mathbb{C} P^{\infty} \cong \operatorname{spf} E^{0}[[t]] .
$$

If we form the elliptic spectrum $\left(E \mathcal{J}_{C}, C, \gamma\right)$, where ${ }^{4}$

$$
E \mathcal{J}_{C}=E \otimes_{\pi_{0} E} \mathcal{J}_{C},
$$

then we have the $\Theta^{2}$-structure

$$
\left(\gamma^{2}\right)^{*} s(C)^{\#} \in C^{2}\left(G_{E}, \mathcal{X}_{C} ; \mathcal{I}(0)\right) .
$$

Theorem 3.7 associates to this $\Theta^{2}$-structure a multiplicative orientation

$$
J_{(E, C, t)}: \mathrm{MSU} \rightarrow E \mathcal{J}_{C} .
$$

Thus we have the following.

7.3 Theorem An elliptic spectrum $(E, C, \gamma)$ determines a canonical map of ring spectra

$$
J_{(E, C, \gamma)}: \mathrm{MSU} \rightarrow E \mathcal{J}_{C} .
$$

Formation of $J_{(E, C, \gamma)}$ is natural, in the sense that if

$$
(f, \alpha):(E, C, \gamma) \rightarrow\left(E^{\prime}, C^{\prime}, \gamma^{\prime}\right)
$$

is a map of elliptic spectra, then the following diagram commutes:

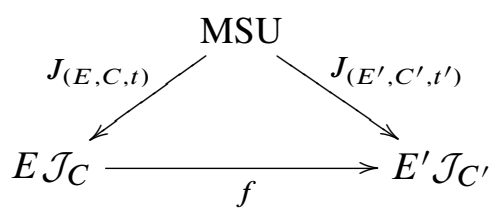

7.4 Definition The Jacobi orientation of the elliptic spectrum $(E, C, \gamma)$ is the map of ring spectra described by the Theorem.

Let $M_{\text {Ell }}$ be the moduli stack of elliptic curves, with universal curve

$$
\pi: \mathcal{C} \rightarrow M_{\text {Ell }}
$$

and identity 0 . Let

$$
\mathcal{J}=\mathcal{J}_{\mathcal{C}}=\underset{k \geq 0}{\operatorname{colim}} \mathcal{I}(0)^{-k}
$$

be the indicated sheaf of algebras on $\mathcal{C}$, and let $\underline{\omega}=0^{*} \mathcal{I}(0)$ be the sheaf on $M_{\text {Ell }}$ of cotangent vectors at the origin in $\mathcal{C}$.

\footnotetext{
${ }^{4}$ A generalized elliptic curve $C / R$ is flat over spec $R$, and so $\mathcal{J}_{C} \cong \Gamma\left(\mathcal{O}_{C \backslash 0}\right)$ is flat over $R$.
} 
7.5 Definition A meromorphic weak Jacobi form of index zero and weight $d$ is a global section of

$$
\mathcal{J} \otimes \pi^{*} \underline{\omega}^{d} .
$$

It is equivalent to give a rule $f$ which associates to each pair $(C / R, \omega)$, consisting of an elliptic curve $C$ over a ring $R$ and a trivialization $\omega$ of $\underline{\omega}_{C}$ a meromorphic function

$$
f(C / R, \omega) \in \mathcal{J}_{C},
$$

subject to the following.

(1) If the following is a pullback diagram

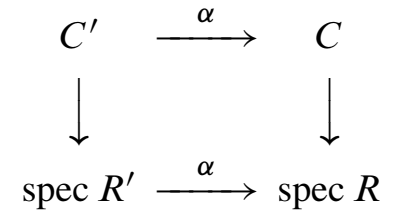

then

$$
f\left(C^{\prime}, \alpha^{*} \omega\right)=\alpha^{*} f(C, \omega) .
$$

(2) If $\lambda \in R^{\times}$then

$$
f(C, \lambda \omega)=\lambda^{-d} f(C, \omega) .
$$

7.6 Remark This sort of Jacobi form, which might be called an arithmetic Jacobi form, was introduced by Kramer [27]. Its relationship to the usual notion of Jacobi form as in [16] is the same as the relationship of the arithmetic to the classical notions of modular form as in [26].

7.7 Theorem If $X$ is an SU-manifold of complex dimension $d$, then as $C$ varies, $J_{(E, C, \gamma)}(X)$ defines a meromorphic Jacobi form of index zero and weight $d$. If $(E, C, \gamma)=K_{\text {Tate }}$, and $y$ is the formal function on the Tate curve corresponding to $u^{-1}$ on $\mathbb{G}_{m}=\operatorname{spec} \mathbb{Z}\left[u, u^{-1}\right]$, then $J_{K_{\text {Tate }}}(X)$ admits an expansion in terms of $y$, and as such

$$
J_{K_{\text {Tate }}}(X)_{y}=\phi(X, q, y)
$$

is the restriction to MSU of the two-variable elliptic genus $\phi$ of (6.5). In particular, $\phi(X, q, y)$ is the $q$-expansion of the meromorphic Jacobi form $J(X)$.

Proof Let $X$ be an SU-manifold of complex dimension $d$. Then

$$
J_{(E, C, \gamma)}(X) \in \pi_{-2 d} E \mathcal{J}_{C} \cong \Gamma\left(\underline{\omega}^{d} \otimes \mathcal{J}_{C}\right),
$$

and so the claim that $J_{(E, C, \gamma)}(X)$ is a Jacobi form follows from the modularity of the Jacobi orientation, together with the fact that $M_{\text {Ell }}$ has a cover by elliptic spectra. 
Alternatively, one can use the argument in the introduction and Section 2.7 of [5] to show that $J_{\text {Tate }}(X)$ is the $q$-expansion of a meromorphic Jacobi form of weight $d$ and index 0 .

The fact that $\left(J_{K_{\text {Tate }}}\right)_{y}$ is the restriction to MSU of the two-variable elliptic genus follows from the fact that, on the one hand, the formula (6.8) is the MSU-characteristic series for the two-variable elliptic genus. On the other hand, (6.8) is also the formula for the cubical structure on a complex elliptic curve of the form $\mathbb{C} / \Lambda$. Indeed in $[5$, Sections 2.6-2.7] this fact is used to show that the $q$-expansion form of $\sigma$ also gives rise to the cubical structure on the Tate elliptic curve over $\mathbb{Z}[[q]]$.

\section{Anomaly cancellation and twists: the Jacobi genus via circle-equivariant elliptic cohomology}

In this section, which is independent of Section 3-Section 7, we show how to obtain the two-variable elliptic genus of $M$ by calculating the $S^{1}$-equivariant Witten genus of $M$, twisted by the tangent bundle of $M$, considered as an $S^{1}$-bundle by the standard action of $S^{1}$ on $T M$. In fact this method also leads to an account of the "level $N$ " genera of $[21 ; 35 ; 22]$.

\subsection{Umkehr maps and genera}

Let $f: X \rightarrow Y$ be a proper map of smooth manifolds. The Becker-Gottlieb-PontrjaginThom construction associates to this situation a stable map

$$
\tau(f): Y_{+} \rightarrow X^{-T f}
$$

where

$$
T f=\operatorname{Ker} d f: T X \rightarrow f^{*} T Y
$$

is the bundle of tangent vectors along the fiber, and $X^{V}$ is the Thom spectrum of the virtual vector bundle $V$.

Associated to any cohomology theory $E$, then, we have a map

$$
\tau(f)^{*}: E^{*} X^{-T f} \rightarrow E^{*} Y .
$$

If the bundle $-T f$ is oriented in $E$-theory, that is, we have a Thom class

$$
U \in E^{-d} X^{-T f},
$$

where $d=\operatorname{rank} T f$, inducing an isomorphism

$$
E^{*} X \cong E^{*-d} X^{-T f}
$$


then the composition of the Thom isomorphism (8.2) with $\tau(f)^{*}$ is the Umkehr homomorphism

$$
f_{!}: E^{*} X \rightarrow E^{*-d} Y \text {. }
$$

We have spelled this out in order to recall the role of the Thom class $U$ in the construction of $f_{!}$, since the notation does not indicate this dependence.

For example, if $X$ is a compact manifold of dimension $d$, and $T=T X$ is its tangent bundle, then associated to the map

$$
\pi^{X}: X \rightarrow *
$$

is the Pontrjagin-Thom map

$$
\tau(\pi): S^{0} \rightarrow X^{-T} .
$$

An $E$-orientation of $-T$ gives an Umkehr map

$$
\pi !: E^{*} X \rightarrow E^{*-d}(*) \text {. }
$$

The class $\pi_{!}(1) \in \pi_{d} E$ is the "genus" of $X$ associated to the orientation $U$. Actually the term genus is appropriate only in the case that the Thom class $U$ is an instance of an exponential family of Thom classes, as we now explain.

To give a map of ring spectra

$$
\phi: \mathrm{MU} \rightarrow E
$$

is equivalent to giving, for every complex vector bundle $V / X$ of rank $d$, an orientation

$$
U_{V} \in E^{d}\left(X^{V}\right)
$$

which is exponential in the sense that

(1) if $\epsilon$ is the trivial bundle of rank 1 , then $U_{\epsilon}$ is the double suspension of 1 in

$$
E^{2}\left(X^{\epsilon}\right)=E^{2}\left(\Sigma^{2} X_{+}\right)
$$

(2) if $V / X$ and $W / Y$ are complex vector bundles of rank $d$ and $e$, then

$$
U_{V \oplus W}=U_{V} \wedge U_{W} \in E^{d+e}\left((X \times Y)^{V \oplus W}\right) \cong E^{d+e}\left(X^{V} \wedge Y^{W}\right) .
$$

The effect of $\phi$ on homotopy groups is a ring homomorphism

$$
\Phi: \operatorname{MU}^{*}\left(S^{0}\right) \rightarrow E^{*}\left(S^{0}\right) \text {. }
$$

The ring $\operatorname{MU}^{*}\left(S^{0}\right)$ is the bordism ring of manifolds $X$ with a complex structure on its stable tangent bundle, and so the ring homomorphism $\Phi$ is a genus.

Let $X$ be such a manifold, of real dimension $2 d$. Thom's theory [33] implies that

$$
\Phi(X)=\pi_{!}^{X}(1) .
$$




\subsection{Twisted genera}

We can apply $\pi_{!}^{X}$ to classes other than 1 . For example, if $\Xi$ is a $E$-characteristic class, then we can define a "twist" of the genus $\Phi$ as

$$
\Phi(X ; \Xi) \stackrel{\text { def }}{=} \pi !(\Xi(T X)) .
$$

Or, if $X$ comes equipped with another vector bundle $V$, then we may form

$$
\Phi(X ; \Xi(V)) \stackrel{\text { def }}{=} \pi_{!}(\Xi(V)) .
$$

\subsection{Anomaly cancellation}

A more interesting situation arises when the bundle $-T$ (or more generally $-T f$ ) does not admit a Thom class. In that case, we may hope to find another vector bundle $V$ on $X$, such that the virtual bundle $V-T f$ does admit a Thom class

$$
U \in E^{*} X^{V-T f} \text {. }
$$

If so, then we may compose the Pontrjagin-Thom map $\tau(f)$ with the zero section

$$
X^{-T f} \rightarrow X^{V-T f}
$$

to obtain a stable map

$$
Y_{+} \rightarrow X^{-T f} \rightarrow X^{V-T f}
$$

We write

$$
f_{!}^{V}: E^{*}(X) \rightarrow E^{*}\left(X^{V-T f}\right) \rightarrow E^{*}\left(X^{-T f}\right) \rightarrow E^{*} Y
$$

for the associated Umkehr map. In the case of the map $\pi^{X}: X \rightarrow *$, we obtain a map

$$
\pi^{X, V}: E^{*}(X) \rightarrow E^{*} S^{0},
$$

and so we have another kind of twisted genus,

$$
\Phi(X ; V)=\pi_{!}^{X, V}(1) .
$$

\subsection{The two-variable elliptic genus as a specialization of the equivariant Witten genus}

Suppose that $V$ is a complex vector bundle, and let $k$ be an integer. The reader interested only in the two-variable genus (6.5) can take $k=-1$ in the following. Let $\mathbb{T}$ be the circle group, let $y^{k}$ be the one-dimensional complex representation of $\mathbb{T}$ in which $w \in \mathbb{T}$ acts as $w^{k}$, and let $z=c_{1}^{\mathbb{T}} y \in H^{2} B \mathbb{T}$. We write $V y^{k}$ for $V$, considered as a $\mathbb{T}$-equivariant vector bundle using the indicated action of $\mathbb{T} \subset \mathbb{C}$. That is,

$$
V y^{k}=V \otimes y^{k} \text {. }
$$


8.6 Lemma If $V$ is a complex vector bundle, then

$$
c_{1}^{\mathbb{T}}\left(V y^{k}-V-(\operatorname{rank} V) y^{k}\right)=0,
$$

and

$$
c_{2}^{\mathbb{T}}\left(V y^{k}-V-(\operatorname{rank} V) y^{k}\right)=-k z c_{1}(V) .
$$

In particular, if $c_{1}(V)=0$, then

$$
c_{2}^{\mathbb{T}}\left(V y^{k}-V-(\operatorname{rank} V) y^{k}\right)=0 .
$$

8.7 Remark The Lemma implies that the map

$$
\mathrm{BSU} \times \mathbb{C} P^{\infty} \rightarrow \mathrm{BSU}
$$

classifying $\xi \otimes(L-1)$ factors through $\mathrm{BU}\langle 6\rangle$. One can see this using connective $K$-theory, $k u$. Note that

$$
\widetilde{k u}^{2 p}(X) \cong[X, \mathrm{BU}\langle 2 p\rangle]
$$

So $L-1$ may be viewed as a class

$$
(L-1) \in \widetilde{k u}^{2}\left(\mathbb{C} P^{\infty}\right),
$$

while the tautological bundle $\xi$ may be viewed as an element of

$$
\xi \in \widetilde{k u}^{4}(\mathrm{BSU}) \text {. }
$$

Thus

$$
\xi \otimes(L-1) \in \widetilde{k u}^{6}\left(\mathrm{BSU} \times \mathbb{C} P^{\infty}\right) \cong\left[\mathrm{BSU} \times \mathbb{C} P^{\infty}, \mathrm{BU}\langle 6\rangle\right] .
$$

The same argument implies that

$$
\prod_{i=1}^{p}\left(1-L_{i}\right) \in \widetilde{k u}^{2 p}\left(\left(\mathbb{C} P^{\infty}\right)^{p}\right) \cong\left[\left(\mathbb{C} P^{\infty}\right)^{p}, \mathrm{BU}\langle 2 p\rangle\right],
$$

which is one of the starting points of [5]. This sheds some light on the relationship between our two approaches to the two-variable elliptic genus.

Proof Let $d=\operatorname{rank}(V)$. Let

$$
c=1+c_{1}+c_{2}+\cdots
$$

denote the total Chern class, and let

$$
c^{\mathbb{T}}=1+c_{1}^{\mathbb{T}}+c_{2}^{\mathbb{T}}+\cdots
$$

denote the total Borel Chern class. If

$$
c(V)=\prod\left(1+x_{i}\right)
$$

Algebraic 83 Geometric Topology, Volume 8 (2008) 
then

$$
\begin{aligned}
c^{\mathbb{T}}\left(V y^{k}\right) & =\prod\left(1+x_{i}+k z\right) \\
c^{\mathbb{T}}\left(d y^{k}\right) & =(1+k z)^{d} \\
c^{\mathbb{T}}\left(V y^{k}-V-d y^{k}\right) & =\prod \frac{1+x_{i}+k z}{\left(1+x_{i}\right)(1+k z)} .
\end{aligned}
$$

Without any assumptions about $c_{1} V$, we have

$$
c_{1}^{\mathbb{T}}\left(V y^{k}\right)=c_{1} V+d k z=c_{1}^{\mathbb{T}}\left(V+d y^{k}\right),
$$

so

$$
c_{1}^{\mathbb{T}}\left(V y^{k}-V-d y^{k}\right)=0 \text {. }
$$

For $c_{2}$, we find that

$$
c_{2}^{\mathbb{T}}\left(V y^{k}\right)=c_{2} V+(d-1) k z c_{1} V+\left(\begin{array}{l}
d \\
2
\end{array}\right) k^{2} z^{2} .
$$

Taking $V$ to be trivial of rank $d$ in (8.8) gives

$$
c_{2}^{\mathbb{T}}\left(d y^{k}\right)=\left(\begin{array}{l}
d \\
2
\end{array}\right) k^{2} z^{2} .
$$

The Whitney sum formula then gives

$$
c_{2}^{\mathbb{T}}\left(V+d y^{k}\right)=c_{2} V+d k z c_{1} V+\left(\begin{array}{l}
d \\
2
\end{array}\right) k^{2} z^{2} .
$$

In general

$$
c_{2}(V-W)=c_{2} V-c_{1} V c_{1} W-c_{2} W+c_{1} W^{2},
$$

which if $c_{1} V=c_{1} W$ simplifies to

$$
c_{2}(V-W)=c_{2} V-c_{2} W .
$$

In our case, this gives

$$
\begin{aligned}
c_{2}^{\mathbb{T}}\left(V y^{k}-V-d y^{k}\right) & =c_{2}(V)+(d-1) k z c_{1} V+\left(\begin{array}{l}
d \\
2
\end{array}\right) k^{2} z^{2} \\
-c_{2} V-d k z c_{1} V-\left(\begin{array}{l}
d \\
2
\end{array}\right) k^{2} z^{2} & \\
& =-k z c_{1} V .
\end{aligned}
$$

We briefly recall some facts about the equivariant elliptic cohomology theory $E=E_{\mathbb{T}}$ of Grojnowski; for more details see Grojnowski [20], Rosu [31], Ando and Basterra [3] 
and Ando [2]. ${ }^{5}$ Let $\Lambda$ be the lattice

$$
\Lambda=2 \pi i \mathbb{Z}+2 \pi i \tau,
$$

and let $C=\mathbb{C} / \Lambda$. For $a \in C$ let

$$
T_{a}: C \rightarrow C
$$

denote translation by $a$. If $X$ is a $\mathbb{T}$-space, then $E_{\mathbb{T}}(X)$ is a sheaf of $\mathbb{Z} / 2$-graded $\mathcal{O}_{\mathbb{C}}$-algebras whose stalk at a point $a \in C$ of exact order $k \leq \infty$ is given by

$$
\left(T_{a}^{*} E_{\mathbb{T}}(X)\right)_{0}=H_{\mathbb{T}}^{*}\left(X^{\mathbb{T}[k]}\right) \otimes_{H^{*} B \mathbb{T}} \mathcal{O}_{C, 0},
$$

where $\mathcal{O}_{C, 0}$ is the stalk of $\mathcal{O}_{C}$ at the identity, and

$$
z \in H^{*} B \mathbb{T} \cong \mathbb{C}[z]
$$

is regarded as an element of $\mathcal{O}_{C, 0}$ via the projection

$$
p: \mathbb{C} \rightarrow C \text {. }
$$

Taking $a=0$ gives

$$
E_{\mathbb{T}}(X)_{0}^{\wedge} \cong H \Lambda_{\mathbb{T}}^{*}(X)
$$

We also recall (see for example Grojnowski [20] or Ando [2, Lemma 7.4]) that if $V$ is a complex $\mathbb{T}$-vector bundle $V$ over a compact $\mathbb{T}$-space $X$, then

$$
E(V) \stackrel{\text { def }}{=} E\left(X^{V}\right)
$$

is an invertible $E(X)$-module.

The main result of [3] and [2] is the construction of a Thom class in $E\left(X^{\xi}\right)$ when $\xi$ is a virtual $\mathbb{T}$-bundle with $c_{1}^{\mathbb{T}} \xi=0=c_{2}^{\mathbb{T}} \xi$. Applied to the current situation, their results give the following.

8.11 Proposition If $c_{1} V=0$, then the bundle $V y^{-1}-V-d y^{-1}$ has a canonical Thom class

$$
U \in \Gamma\left(E\left(V y^{-1}\right) \otimes E(V)^{-1} \otimes E\left(y^{-1}\right)^{-d}\right),
$$

whose value in the stalk at the origin is the Borel-equivariant Thom class given by the sigma orientation of $V y^{-1}-V-d y^{-1}$.

\footnotetext{
${ }^{5}$ One can carry out the analysis in this section using Greenlees's equivariant elliptic cohomology [18]; the necessary prerequisites are the subject of [4].
}

Algebraic $8 \mathcal{G}$ Geometric Topology, Volume 8 (2008) 
In particular, if $V=T=T X$ is the rank- $d$ complex tangent bundle of a compact manifold $X$, then we may consider the composition

$$
S^{0} \rightarrow X^{-T} \rightarrow X^{T y^{-1}-T},
$$

where the first map is the Pontrjagin-Thom map, and the second is the relative zero section. After desuspending by $d y^{-1}$ this gives

$$
g: S^{-d y^{-1}} \rightarrow X^{-T-d y^{-1}} \rightarrow X^{T y^{-1}-T-d y^{-1}} .
$$

If $c_{1} X=0$, then Proposition 8.11 gives a class

$$
U \in E\left(X^{T y^{-1}-T-d y^{-1}}\right),
$$

which we may pull back along $g$. Recall the following.

$$
\begin{aligned}
& \text { 8.13 Lemma } \quad E\left(S^{y^{-1}}\right)=\mathcal{I}(0) \text {, } \\
& \text { and so } \quad E\left(S^{-d y^{-1}}\right)=\mathcal{I}(0)^{-d}
\end{aligned}
$$

Proof It's illuminating to give two proofs. First, consider the cofiber sequence of $\mathbb{T}$-spaces

$$
\mathbb{C}^{\times} \rightarrow \mathbb{C} \rightarrow S^{y^{-1}} .
$$

Let $S=\operatorname{spec} \mathbb{C}$, and let $\pi$ be the structure map

$$
\pi: C \rightarrow S \text {. }
$$

It's easy to check using (8.10) that we have a commutative diagram

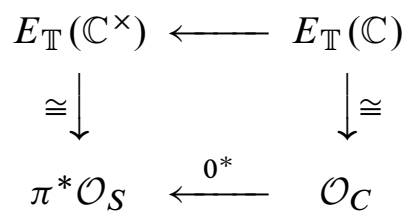

in which the vertical arrows are isomorphism as indicated. It follows that

$$
E_{\mathbb{T}}\left(S^{y^{-1}}\right) \cong \mathcal{I}(0),
$$

and the general case follows by taking tensor powers.

Alternatively, observe that (8.10) gives

$$
\left(T_{a}^{*} E_{\mathbb{T}}\left(S^{y^{-1}}\right)\right)_{0} \cong \mathcal{O}_{C, 0}
$$


for $a \neq 0$, while, letting $L$ denote the line bundle over $\mathbb{C} P^{\infty}$ corresponding to the representation $y^{-1}$,

$$
E_{\mathbb{T}}\left(S^{y^{-1}}\right)_{0} \cong H^{*}\left(\left(\mathbb{C} P^{\infty}\right)^{L}\right) \otimes \mathcal{O}_{C, 0} \cong \mathcal{I}(0)_{0} .
$$

This is a description of the line bundle $\mathcal{I}(0)$.

Let

$$
\mathcal{E}(X) \stackrel{\text { def }}{=} \pi_{!}^{T, T y^{-1}-d y^{-1}}(1)=g^{*} U \in E\left(S^{-d y^{-1}}\right)=\Gamma \mathcal{I}(0)^{-d} .
$$

\subsection{Proposition We have}

$$
\mathcal{E}(X)=J_{(H \Lambda, \mathbb{C} / \Lambda, \widehat{p})}(X)=\phi\left(X, y^{-1}, q\right),
$$

where $\phi$ is the two-variable genus of Equation (6.5).

Proof Let

$$
c(T)=\prod_{i=1}^{d}\left(1+x_{i}\right) .
$$

Let $w$ be a point of $C$, and suppose that $z \in \mathbb{C}$ is such that $p(z)=w$. According to [3, Section 6.2] or [2, Section 8.2], the class $U$ at $w$ is the Thom class associated to the Euler class

$$
\prod_{i=1}^{d} \frac{\sigma\left(x_{i}-z, \tau\right)}{\sigma\left(x_{i}, \tau\right) \sigma(-z, \tau)},
$$

and we have already shown that this is the Euler class associated to the genus $\phi\left(M, y^{-1}, q\right)$.

\subsection{Level $N$ genera}

If we know only that $c_{1} X \equiv 0 \bmod N$, then Lemma 8.6 shows that

$$
c_{2}^{\mathbb{T}[N]}\left(T y^{-1}-T-d y^{-1}\right)=0 .
$$

Noting that

$$
E \mathbb{T} \times_{\mathbb{T}} \mathbb{T} \times_{\mathbb{T}[N]} X \cong E \mathbb{Z} / n \times_{\mathbb{T}[N]} X,
$$

we make the following definition. Again let $E_{\mathbb{T}}$ be Grojnowski's equivariant elliptic cohomology, associated to the complex curve $C=\mathbb{C} / \Lambda$. 
8.17 Definition Let $X$ be an $C$-space. We define the $\mathbb{T}[N]$-equivariant elliptic cohomology of $X$ to be

$$
E_{\mathbb{T}[N]}(X)=E_{\mathbb{T}}\left(\mathbb{T} \times_{\mathbb{T}[N]} X\right),
$$

where $\mathbb{T} \times_{\mathbb{T}[N]} X$ is considered as a $\mathbb{T}$ space by acting only on the left of $\mathbb{T}$.

We recall the following lemma:

$$
\text { 8.18 Lemma } \quad E_{\mathbb{T}[N]}(*)=\mathcal{O}_{C[N]} .
$$

More generally, if $X$ is a $\mathbb{T}[N]$-space, then

$$
E_{\mathbb{T}[N]}(X)_{a}=0
$$

unless $a \in C[N]$, and if $a$ has exact order $k$ dividing $N$, then

$$
T_{a}^{*} E_{\mathbb{T}[N]}(X)_{a} \cong H^{*} X^{\mathbb{T}[k]}
$$

and the map

$$
T_{a}^{*} E_{\mathbb{T}}\left(\mathbb{T} \times_{\mathbb{T}[N]} X\right)_{a} \cong H_{\mathbb{T}}\left(\mathbb{T} \times_{\mathbb{T}[N]} X^{\mathbb{T}[k]}\right) \rightarrow H^{*} X^{\mathbb{T}[k]}
$$

corresponds to setting $z=0$ in (8.10).

Proof The stalk of $E_{\mathbb{T}}(Y)$ at a point $a$ of exact order $k \leq \infty$ is

$$
T_{a}^{*} E_{\mathbb{T}}(Y)_{a}=H_{\mathbb{T}}^{*}\left(Y^{\mathbb{T}[k]}\right) \otimes_{H^{*} B \mathbb{T}} \mathcal{O}_{C, 0} .
$$

If $Y=\mathbb{T} \times_{\mathbb{T}[N]} X$ then $Y^{\mathbb{T}[k]}$ is empty unless $k \mid N$, and then

$$
H_{\mathbb{T}}^{*}\left(Y^{\mathbb{T}[k]}\right)=H^{*}\left(B \mathbb{T}[N] \times X^{\mathbb{T}[k]}\right)=H^{*}\left(X^{\mathbb{T}[k]}\right)
$$

(recall that we are working with complex coefficients).

We still have

$$
\begin{aligned}
& c_{1}^{\mathbb{T}}\left(\mathbb{T} \times_{\mathbb{T}[N]}(T y-T-d y)\right)=0 \\
& c_{2}^{\mathbb{T}}\left(\mathbb{T} \times_{\mathbb{T}[N]}(T y-T-d y)\right)=0
\end{aligned}
$$

and so now [3] and [2] imply:

8.20 Proposition The bundle $T y^{-1}-T-d y^{-1}$ has a canonical Thom class

$$
U_{N} \in\left(E_{\mathbb{T}[N]}\left(T y^{-1}\right) \otimes E_{\mathbb{T}[N]}(T)^{-1} \otimes E_{\mathbb{T}[N]}\left(y^{-1}\right)^{-d}\right) .
$$


We can now define

$$
\mathcal{E}_{N}(X)=g^{*} U_{N} \in \Gamma E_{\mathbb{T}[N]}\left(S^{-d y^{-1}}\right) .
$$

where, as in (8.12), $g$ is the map

$$
g: S^{-d y^{-1}} \rightarrow X^{-T-d y^{-1}} \rightarrow X^{T y^{-1}-T-d y^{-1}} .
$$

Now

$$
\left(S^{-y}\right)^{\mathbb{T}[N]}=S^{0}
$$

so Lemma 8.18 implies that

$$
E_{\mathbb{T}[N]}\left(S^{-d y^{-1}}\right) \cong \mathcal{O}_{C[N]}
$$

8.21 Proposition The value of $\mathcal{E}_{N}(X)$ at a point $a \in C[N]$ is the level-N genus of $X$, as in $[21 ; 35]$, evaluated at $a$.

Proof The recipe for calculating $g^{*} U_{N}$ at $a \in C[N]$ is the following [20]. Let's write $W$ for our bundle

$$
W=T y^{-1}-T-d y^{-1} .
$$

Recall that $T_{a}$ is the translation map

$$
T_{a}: C \rightarrow C .
$$

The construction of $E_{\mathbb{T}}$ is such that

$$
T_{a}^{*} U_{N} \in H_{\mathbb{T}[N]}^{*}\left(M^{W^{A}}\right) .
$$

We may calculate

$$
g^{*} T_{a}^{*} U_{N} \in H_{\mathbb{T}[N]}^{*}(*)=\mathbb{C}
$$

using classical techniques, and this is the value of $\mathcal{E}_{N}$ at $a$.

Let $a=\frac{2 \pi i}{N}(l+k \tau)$, with $0 \leq k \leq N-1$. According to [2, Section 9], $T_{a}^{*} U_{N}$ is the class in

$$
H_{\mathbb{T}}^{*}\left(\left(\mathbb{T} \times_{\mathbb{T}[N]} X^{W}\right)^{\mathbb{T}[N]}\right) \otimes \mathcal{O}_{C, 0} \cong H_{\mathbb{T}[N]}^{*}\left(\left(\mathbb{T} \times_{\mathbb{T}[N]} X^{W}\right)^{\mathbb{T}[N]}\right)
$$

whose Euler class in

$$
H_{\mathbb{T}}^{*}\left(\left(\mathbb{T} \times_{\mathbb{T}[N]} X\right)^{\mathbb{T}[N]}\right) \otimes \mathcal{O}_{C, 0}
$$

is equal to

$$
\exp \left(-\frac{k}{N} \sum x_{i}\right) \prod_{i} \frac{\sigma\left(x_{i}-z-a\right)}{\sigma\left(x_{i}\right) \sigma(-z-a)}
$$

Algebraic 83 Geometric Topology, Volume 8 (2008) 
By definition, the quantity $g^{*} T_{a}^{*} U$ in $(\mathbf{8 . 2 2})$ is the genus associated to this expression, with $z=0$. Now observe that setting $z=0$ in $(\mathbf{8 . 2 3})$ gives the Euler class associated to the level- $N$ genus.

\section{References}

[1] J F Adams, Stable homotopy and generalised homology, Univ. of Chicago Press (1974) MR0402720Chicago Lectures in Math.

[2] M Ando, The sigma orientation for analytic circle-equivariant elliptic cohomology, Geom. Topol. 7 (2003) 91-153 MR1988282

[3] M Ando, M Basterra, The Witten genus and equivariant elliptic cohomology, Math. Z. 240 (2002) 787-822 MR1922730

[4] M Ando, JPC Greenlees, Circle-equivariant classifying spaces and the rational equivariant sigma genus arXiv:abs/0705.2687Submitted

[5] M Ando, MJ Hopkins, N P Strickland, Elliptic spectra, the Witten genus and the theorem of the cube, Invent. Math. 146 (2001) 595-687 MR1869850

[6] L Borisov, A Libgober, Elliptic genera of singular varieties, orbifold elliptic genus and chiral de Rham complex, from: "Mirror symmetry, IV (Montreal, QC, 2000)", AMS/IP Stud. Adv. Math. 33, Amer. Math. Soc. (2002) 325-342 MR1969036

[7] L Borisov, A Libgober, Elliptic genera of singular varieties, Duke Math. J. 116 (2003) 319-351 MR1953295

[8] L Borisov, A Libgober, McKay correspondence for elliptic genera, Ann. of Math. (2) 161 (2005) 1521-1569 MR2180406

[9] L Breen, Fonctions thêta et théorème du cube, Lecture Notes in Mathematics 980, Springer, Berlin (1983) MR823233

[10] R L Cohen, J D S Jones, G B Segal, Floer's infinite-dimensional Morse theory and homotopy theory, from: "The Floer memorial volume", Progr. Math. 133, Birkhäuser, Basel (1995) 297-325 MR1362832

[11] P Deligne, Courbes elliptiques: formulaire d'après J. Tate, from: "Modular functions of one variable, IV (Proc. Internat. Summer School, Univ. Antwerp, Antwerp, 1972)”, Lecture Notes in Math. 476, Springer, Berlin (1975) 53-73 MR0387292

[12] P Deligne, M Rapoport, Les schémas de modules de courbes elliptiques, from: "Modular functions of one variable, II (Proc. Internat. Summer School, Univ. Antwerp, Antwerp, 1972)", Springer, Berlin (1973) 143-316. Lecture Notes in Math., Vol. 349 MR0337993

[13] R Dijkgraaf, G Moore, E Verlinde, H Verlinde, Elliptic genera of symmetric products and second quantized strings, Comm. Math. Phys. 185 (1997) 197-209 MR1463039 
[14] E Dyer, Cohomology theories, Mathematics Lecture Note Series, W. A. Benjamin, New York-Amsterdam (1969) MR0268883

[15] H Eguchi, H Ooguri, A Taormina, S-K Yang, Superconformal algebras and string compactification on manifolds with SU(N) holonomy, Nucl. Phys. B 315 (1989) 193-291

[16] M Eichler, D Zagier, The theory of Jacobi forms, Progress in Mathematics 55, Birkhäuser, Boston (1985) MR781735

[17] N Ganter, Orbifold genera, product formulas and power operations, Adv. Math. 205 (2006) 84-133 MR2254309

[18] J P C Greenlees, Rational $S^{1}$-equivariant elliptic cohomology, Topology 44 (2005) 1213-1279 MR2168575

[19] J P C Greenlees, J P May, Generalized Tate cohomology, Mem. Amer. Math. Soc. 113 (1995) viii+178 MR1230773

[20] I Grojnowski, Delocalized equivariant elliptic cohomology, from: "Elliptic cohomology", (H R Miller, D C Ravenel, editors), London Math. Society Lecture Note Series 342, Cambridge University Press (2007) xii+364 MR2330502Geometry, applications, and higher chromatic analogues, Papers from the Workshop on Elliptic Cohomology and Chromatic Phenomena held in Cambridge, December 9-20, 2002,

[21] F Hirzebruch, Elliptic genera of level $N$ for complex manifolds, from: "Differential geometrical methods in theoretical physics (Como, 1987)", NATO Adv. Sci. Inst. Ser. C Math. Phys. Sci. 250, Kluwer Acad. Publ., Dordrecht (1988) 37-63 MR981372

[22] F Hirzebruch, T Berger, R Jung, Manifolds and modular forms, Aspects of Mathematics E20, Friedr. Vieweg \& Sohn, Braunschweig (1992) MR1189136With appendices by Nils-Peter Skoruppa and by Paul Baum

[23] G Höhn, Komplexe elliptische Geschlechter und $S^{1}$-äquivariante Kobordimustheorie (complex elliptic genera and $S^{1}$-equivariant cobordism theory), Diplomarbeit, Bonn (1991) arXiv:math.AT/0405232

[24] M J Hopkins, Topological modular forms, the Witten genus, and the theorem of the cube, from: "Proceedings of the International Congress of Mathematicians, Vol. 1, 2 (Zürich, 1994)”, Birkhäuser, Basel (1995) 554-565 MR1403956

[25] MJ Hopkins, Algebraic topology and modular forms, from: "Proceedings of the International Congress of Mathematicians, Vol. I (Beijing, 2002)", Higher Ed. Press, Beijing (2002) 291-317 MR1989190

[26] N M Katz, p-adic properties of modular schemes and modular forms, from: "Modular functions of one variable, III (Proc. Internat. Summer School, Univ. Antwerp, Antwerp, 1972)", Springer, Berlin (1973) 69-190. Lecture Notes in Mathematics, Vol. 350 MR0447119 
[27] J Kramer, An arithmetic theory of Jacobi forms in higher dimensions, J. Reine Angew. Math. 458 (1995) 157-182 MR1310957

[28] I M Krichever, Generalized elliptic genera and Baker-Akhiezer functions, Mat. Zametki 47 (1990) 34-45, 158 MR1048541

[29] L G Lewis, Jr, JP May, M Steinberger, J E McClure, Equivariant stable homotopy theory, Lecture Notes in Math. 1213, Springer, Berlin (1986) MR866482With contributions by J. E. McClure

[30] J E McClure, $E_{\infty}$-ring structures for Tate spectra, Proc. Amer. Math. Soc. 124 (1996) 1917-1922 MR1307549

[31] I Rosu, Equivariant elliptic cohomology and rigidity, Amer. J. Math. 123 (2001) 647677 MR1844573

[32] Y B Rudyak, On Thom spectra, orientability, and cobordism, Springer Monographs in Mathematics, Springer, Berlin (1998) MR1627486With a foreword by Haynes Miller

[33] R Thom, Quelques propriétés globales des variétés différentiables, Comment. Math. Helv. 28 (1954) 17-86 MR0061823

[34] E Witten, Elliptic genera and quantum field theory, Comm. Math. Phys. 109 (1987) 525-536 MR885560

[35] E Witten, The index of the Dirac operator in loop space, from: "Elliptic curves and modular forms in algebraic topology (Princeton, NJ, 1986)", Lecture Notes in Math. 1326, Springer, Berlin (1988) 161-181 MR970288

Department of Mathematics, The University of Illinois at Urbana-Champaign

Urbana IL 61801, USA

Department of Mathematics and Statistics, Grinnell College

Grinnell IA 50112, USA

Department of Mathematics, Colby College

Waterville ME 04901, USA

mando@math.uiuc.edu, frenchc@math.grinnell.edu, ganter@math.uiuc.edu

Received: 16 June $2006 \quad$ Revised: 8 November 2007 